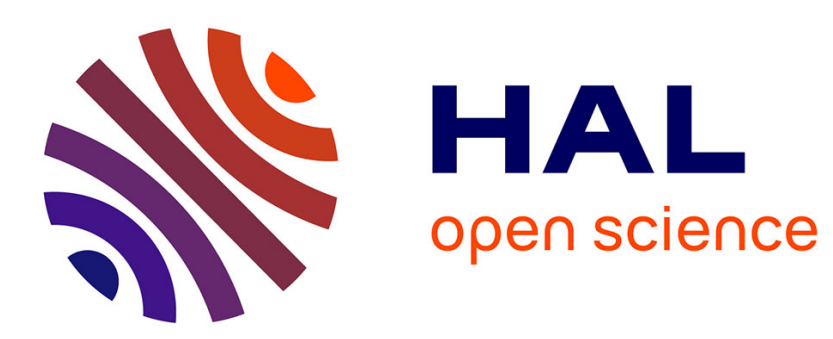

\title{
Modeling and validation of the elasticity parameters of multi-layer specimens pertinent to silicone vocal fold replicas
}

Mohammad Ahmad, Anne Bouvet, Xavier Pelorson, Annemie van Hirtum

\section{- To cite this version:}

Mohammad Ahmad, Anne Bouvet, Xavier Pelorson, Annemie van Hirtum. Modeling and validation of the elasticity parameters of multi-layer specimens pertinent to silicone vocal fold replicas. International Journal of Mechanical Sciences, 2021, 208, pp.106685. 10.1016/j.ijmecsci.2021.106685 . hal-03325889

\section{HAL Id: hal-03325889 \\ https://hal.science/hal-03325889}

Submitted on 25 Aug 2021

HAL is a multi-disciplinary open access archive for the deposit and dissemination of scientific research documents, whether they are published or not. The documents may come from teaching and research institutions in France or abroad, or from public or private research centers.
L'archive ouverte pluridisciplinaire HAL, est destinée au dépôt et à la diffusion de documents scientifiques de niveau recherche, publiés ou non, émanant des établissements d'enseignement et de recherche français ou étrangers, des laboratoires publics ou privés. 


\title{
Modeling and validation of the elasticity parameters of multi-layer specimens pertinent to silicone vocal fold replicas
}

Mohammad Ahmad, Anne Bouvet, Xavier Pelorson, Annemie Van Hirtum ${ }^{1}$

LEGI, UMR CNRS 5519, Grenoble Alpes University, France

\begin{abstract}
Deformable silicone-molded multi-layer vocal folds replicas are commonly used in physical studies of vocal folds auto-oscillation. Nevertheless, few studies deal with the reproducibility or the mechanical properties of silicone moldings. In the current study, the effective elastic Young's modulus of molded silicone bone-shaped specimens consisting of parallel and serial stacked layers is sought. The Young's modulus of each layer is pertinent for one of the anatomical vocal folds layers whereas layer dimensions are designed so that the dimension ratio between adjacent layers varies in the range observed on human vocal folds. The effective Young's modulus for onelayer, two-layer and three-layer specimens is then determined experimentally (between $4 \mathrm{kPa}$ and $65 \mathrm{kPa}$ ) with uni-axial mechanical press tests or with precision loading tests so that both methods are cross-validated (difference less than $3.5 \mathrm{kPa}$ ). The overall layer dimension molding accuracy $(1.5 \mathrm{~mm})$ has no significant impact (less than $1.2 \mathrm{kPa}$ ) on the sought effective Young's modulus and the molding procedure is considered reproducible. Furthermore, an analytical model approach for multi-layer stacked specimen is validated (overall accuracy of $2.4 \mathrm{kPa}$ ) against the experimental data.
\end{abstract}

Keywords: Multi-layer silicone-molded stacked specimen, Uni-axial stress testing, Effective Young's modulus, Analytical model, Small strain range

\section{Nomenclature}

\footnotetext{
${ }^{1}$ Corresponding author: annemie.vanhirtum@univ-grenoble-alpes.fr
} 
$\Delta h, \Delta l$ difference between the measured and designed dimension

$\Delta l \quad$ elongation parallel to the force direction

$\mathcal{F} \quad$ uni-axial force

${ }_{5} l \quad$ length parallel to the force direction

$l_{0} \quad$ initial length

$\mathcal{M}$ mass mixing ratio

$\mathcal{B}_{(j, j+1)}$ composition ratio between the Young's moduli of adjacent layers

$\mathcal{H}_{(j, j+1)}$ height stacking dimension ratio between adjacent layers

${ }_{10} \mathcal{L}_{(j, j+1)}$ length stacking dimension ratio between adjacent layers

$\nabla_{(j, j+1)} \in\left\{\mathcal{H}_{(j, j+1)}, \mathcal{L}_{(j, j+1)}\right\}$ stacking dimension ratio

$\mathcal{V} \quad$ volume

|| parallel stacking

$\perp \| \quad$ combined serial and parallel stacking

$15 \quad \perp \quad$ serial stacking

$\rho \quad$ density

$\mathcal{A} \quad$ cross-section area

$\mathcal{A}^{*} \quad$ cross-section area based on volume conservation

$\mathcal{A}^{q} \quad$ cross-section area based on a quadratic fit

${ }_{20} \mathcal{A}_{0} \quad$ initial area

$\varepsilon \quad$ deformation strain

$\sigma \quad$ stress

$\xi \quad$ relative discrepancy

$\mathcal{E} \quad$ Young's modulus 
$\mathcal{E}_{\text {eff }}^{d} \quad$ modelled $\mathcal{E}_{\text {eff }}$ for design composites

$\mathcal{E}_{\text {eff }}^{M P} \quad$ measured $\mathcal{E}_{\text {eff }}$ with the mechanical press

$\mathcal{E}_{\text {eff }}^{P L} \quad$ measured $\mathcal{E}_{\text {eff }}$ with precision loading

$\mathcal{E}_{\text {eff }}^{r e f} \quad$ literature value of $\mathcal{E}_{\text {eff }}$ for $1 \mathrm{~L}$ mixtures

30 $\mathcal{E}_{\text {eff }}^{s-M P}$ modelled specimen $\mathcal{E}_{\text {eff }}$ with measured layer $\mathcal{E}_{\text {eff }}^{M P}$

$\mathcal{E}_{\text {eff }}^{s-P L}$ modelled specimen $\mathcal{E}_{\text {eff }}$ with measured layer $\mathcal{E}_{\text {eff }}^{P L}$

$\mathcal{E}_{\text {eff }}^{s-r e f}$ modelled specimen $\mathcal{E}_{\text {eff }}$ with reference layer $\mathcal{E}_{\text {eff }}^{\text {ref }}$

d superscript indicating design dimensions

$g_{0} \quad$ gravitational constant

$35 h$ height perpendicular to the force direction

$i=1 \ldots n, j=1 \ldots n-1$ layer indices

$m \quad$ mass

$n$ total number of layers in a composite

$o_{(j, j+1)} \in\{\perp, \|\}$ stacking orientation between adjacent layers

$40 \quad R \quad$ coefficient of determination

$r \quad$ relative mass portion

ref superscript indicating reference values obtained from literature

$s \quad$ superscript indicating measured dimensions

$t \quad$ subscript indicating true value (for stress and strain)

${ }_{45} w \quad$ constant layer width of $15 \mathrm{~mm}$

$x \quad$ position along the force direction 
1L, 2L, 3L, 4L one-layer, two-layer, three-layer, four-layer

I one-layer specimen, $n=1$

II two-layer specimen, $n=2$

50 III three-layer specimen, $n=3$

D, (-)D silicone Dragonskin

E, (-)E silicone Ecoflex

M5, MRI, EPI labels of three silicone VF replicas

ML multi-layer

55 MP mechanical press

PL precision loading

T, T(-) silicone Thinner

TD mixture of silicone Thinner and Dragonskin

TE mixture of silicone Thinner and Ecoflex

${ }_{60}$ VF vocal fold

WAM weighted arithmetic mean

WHM weighted harmonic mean

\section{Introduction}

Physical studies of phonation or vocal fold (VF) auto-oscillation often rely on deformable mechanical VF replicas to assess the fluid-structure interaction in the larynx between the airflow coming from the lungs and the surrounding VF tissues. One major criterion for silicone VF replica design consists in mimicking, to some extent, the complex anatomical structure of a human VF illustrated in Fig 1(a), This results in multi-layer (ML) representations 70 of a human VF such as the one depicted in Fig. 1(b). Deformable silicone mechanical VF replicas, mimicking the ML representation of a human VF, 


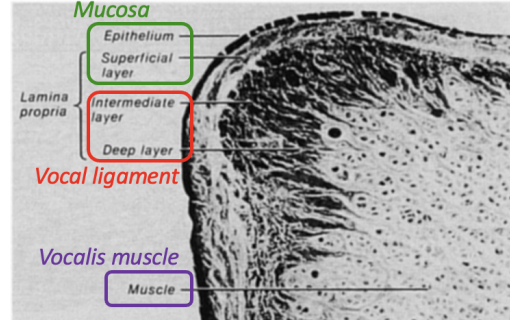

(a) ex-vivo observation

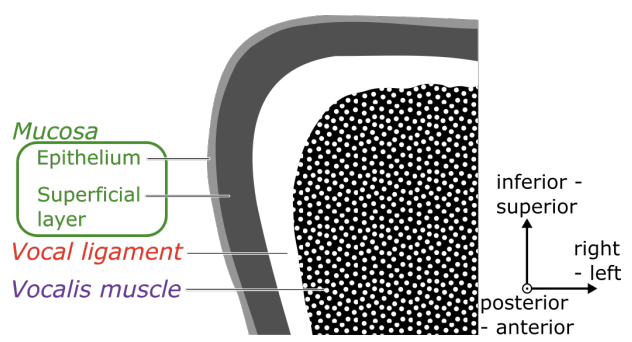

(b) a multi-layer representation

Figure 1: Illustration of the anatomical structure of a left human VF in the medio-frontal plane [5]: a) realistic coronal section (adapted from [6]), b) example of a multi-layer representation (adapted from [7]).

are then obtained as an overlap of silicone molding layers [1, 2, 3, 4].

Fig. 2 illustrates three deformable multi-layered (ML) silicone VF repli75 cas [3, 8]. The M5 replica follows an anatomical two-layer (2L) body-cover representation including the vocalis muscle and the superficial layer [9]. This $2 \mathrm{~L}$ representation is mainly motivated by the mucosal wave theory of normal $\mathrm{VF}$ vibration, which situates vibration within the mucosal layers only [10]. The MRI replica has an anatomical three-layer (3L) structure by adding a third thin surface layer representing the epithelium to the $2 \mathrm{~L}$ structure of the M5 replica [1, 4]. The EPI replica is an anatomical four-layer (4L) structure obtained by inserting an extremely soft deep layer between the muscle and the superficial layer of the 3L structure of the MRI replica [2]. Physical studies [4, 8, have shown that these VF replicas allow one to reproduce sustained VF auto-oscillation. Nevertheless, major flow and vibration properties, such as their auto-oscillation frequency and required minimum upstream pressure (phonation onset threshold), vary between replicas. These differences are partly attributed to the elasticity of the replicas, which depend on the adopted VF representation and the layer molding. Despite its role in the fluid-structure interaction, and hence on observed feature values and physical model validation, the effective elasticity of silicone VF replicas is rarely considered, instead the elasticity of the individual molding layers is mentioned.

Each molding layer composition within the silicone VF replicas is assumed to be an elastic and isotropic solid material consisting of a single constituent 


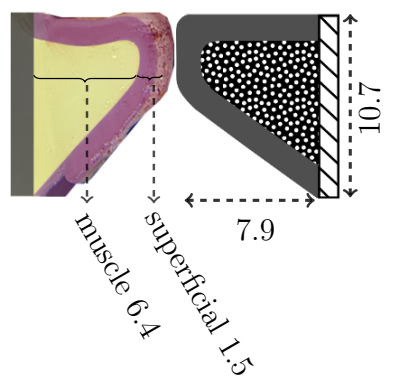

(a) M5

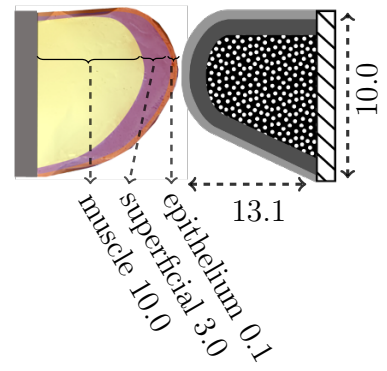

(b) MRI

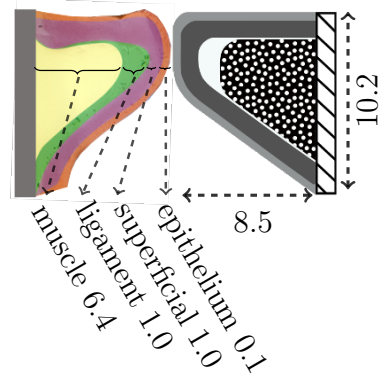

(c) EPI

Figure 2: Coronal section (dimensions in $\mathrm{mm}$ ) of a molded silicone VF replica layers (right VF) and its schematic multi-layer representation (left VF) [3, 8, 7]: a) M5, b) MRI and c) EPI. For illustrative purposes different layers of the right VF were molded with different colors.

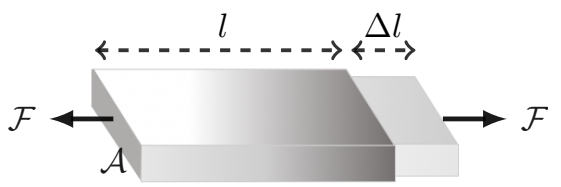

Figure 3: Elastic material with cross-section $\mathcal{A}$, length $l$ and elongation $\Delta l$ following an uni-axial force $\mathcal{F}$. 
or of a mixture of multiple constituents. The layers elasticity is then characterised by Young's modulus $\mathcal{E}$ for a single constituent (or component) and by effective Young's modulus $\mathcal{E}_{\text {eff }}$ for a mixture of constituents (or components). Considering a material portion with length $l$ and cross-section $\mathcal{A}$ depicted in Fig. 3. Young's modulus $\mathcal{E}_{(e f f)}$ relates stress $\sigma$,

$$
\sigma=\frac{\mathcal{F}}{\mathcal{A}}
$$

exerted by an uni-axial force $\mathcal{F}$, to its relative linear deformation strain $\varepsilon$,

$$
\varepsilon=\frac{\Delta l}{l}
$$

expressing a linear stress-strain relationship

$$
\mathcal{E}_{(e f f)}=\frac{\sigma}{\varepsilon}
$$

95

with $\Delta l \geq 0$ denoting the elongation along the force $\mathcal{F}$ direction.

The aim of this work is to consider and validate an analytical model for the effective Young's modulus $\mathcal{E}_{\text {eff }}$ of ML silicone composites from its layers properties, i.e. $\mathcal{E}_{\text {eff }}$ and geometry. A validated analytical model predicting $\mathcal{E}_{\text {eff }}$ of ML moldings is of interest for the (a-priori) mechanical characterisation, and eventually the design of ML VF representations, for normal as well as for abnormal VF structures. Indeed, in the long term, such a model is of particular interest for physical studies (using deformable silicone-based molded replicas) involving a systematic elasticity variation mimicking either intra- and inter-speaker diversity (voice type, morphology, aging etc. [11]) or a structural pathology (scar, nodule, carcinoma, cyst etc. [5]). In addition, the reproducibility of silicone moldings in terms of $\mathcal{E}_{\text {eff }}$ is sought.

\section{Overview of layer compositions in silicone VF replicas}

Each molding layer of the silicone VF replicas illustrated in Fig. 2 is a different mixture of silicone thinner and Ecoflex (TE, silicone Thinner and twopart A\&B Ecoflex 00-30, Smooth-On, Inc., Easton, PA) or silicone thinner and Dragonskin (TD, silicone Thinner and two-part A \& fast B Dragon Skin 
Table 1: VF layer properties for a male adult [12, 13, 14, 15, 16, 2, 17, 18, 19] and silicone replicas $\left(\mathrm{M} 5^{\star}, \mathrm{MRI}^{\dagger}, \mathrm{EPI}^{\ddagger}[8]\right)$ : Young's modulus $\mathcal{E}[\mathrm{kPa}$ for human $\mathrm{VF}$ anatomical layers, effective Young's modulus of mixtures in replica layers $\mathcal{E}_{\text {eff } f}[\mathrm{kPa}]$, mixtures TE (ThinnerEcoflex) or TD (Thinner-Dragonskin) and mixing ratio $\mathcal{M}$.

\begin{tabular}{|c|c|c|c|c|}
\hline \multirow[b]{2}{*}{ Layer } & Adult $\|$ & \multicolumn{3}{|c|}{ VF replica } \\
\hline & $\mathcal{E}[\mathrm{kPa}] \|$ & $\mathcal{E}_{e f f}[\mathrm{kPa}]$ & Mixture & $\mathcal{M}[-]$ \\
\hline Muscle & $8-29^{\star \star}$ & $10.4^{\star} 4.9^{\dagger} 21.9^{\ddagger}$ & $\mathrm{TE}$ & $2: 2^{\star} 4: 2^{\dagger} 1: 2^{\ddagger}$ \\
\hline Ligament & $10-45$ & $4.9^{\ddagger}$ & $\mathrm{TE}$ & $4: 2^{\ddagger}$ \\
\hline Superficial & $2-9$ & $4.9^{\star} 0.2^{\dagger, \ddagger}$ & $\mathrm{TE}$ & $4: 2^{\star} 8: 2^{\dagger, \ddagger}$ \\
\hline Epithelium & $40-60$ & $52^{\dagger, \ddagger}$ & $\mathrm{TD}$ & $1: 2^{\dagger, \ddagger}$ \\
\hline
\end{tabular}

10, Smooth-On, Inc., Easton, PA). The mass mixing ratio $\mathcal{M}=r_{T}: r_{E(D)}$ for each TE (or TD) mixture expresses the relative mass of silicone thinner to Ecoflex (or Dragonskin). The relative mass portion of silicone thinner $r_{T}$ is varied between 1 and 8 whereas the relative mass portion of two-part Ecoflex (or Dragonskin) is held constant $r_{E(D)}=2$ (1 part A and 1 part B).

Used mixtures (TE or TD), mixing ratios $\mathcal{M}$ and Young's moduli $\mathcal{E}_{(e f f)}$ for each layer in the M5, MRI and EPI replicas shown in Fig. 2, are detailed in Table 1 [3, 8]. Young's moduli $\mathcal{E}_{\text {eff }}$ (up to $52 \mathrm{kPa}$ ) are consistent with the order of magnitudes (up to $60 \mathrm{kPa}$ ) associated with the anatomical layers of a human VF [12, 13, 14, 15, 16, 2, 17, 18, 19]. Layer thicknesses of silicone VF replicas indicated in Fig. 2 are of the same order of magnitude as those associated with a human VF [ 8,3$]$. The ratio of layer thicknesses with respect to the molding layer representing the superficial layer varies from 0.1 up to 6.4, i.e. 4.3 for M5, between 3.3 and 0.03 for MRI and between 6.4 and 0.1 for EPI. For all replicas the molding layer representing the muscle is thicker and has a higher Young's modulus than the molding layer representing the superficial layer.

A literature overview of Young's moduli $\mathcal{E}_{(e f f)}$ and densities $\rho$ for TE and TD mixtures is given in Table 2 [1, 2, 20, 21, 22]. Individual components (()D, (-)E and T(-)) are denoted using the TD and TE mixture notation where 
Table 2: Material properties for single components ((-)D, (-)E and $\mathrm{T}(-))$ and mixtures (TD and TE) reported in literature: Young's modulus $\mathcal{E}[\mathrm{kPa}]$ for components and $\mathcal{E}_{\text {eff }}$ $[\mathrm{kPa}]$ for their mixtures and density $\rho\left[\mathrm{kg} \cdot \mathrm{m}^{-3}\right]$.

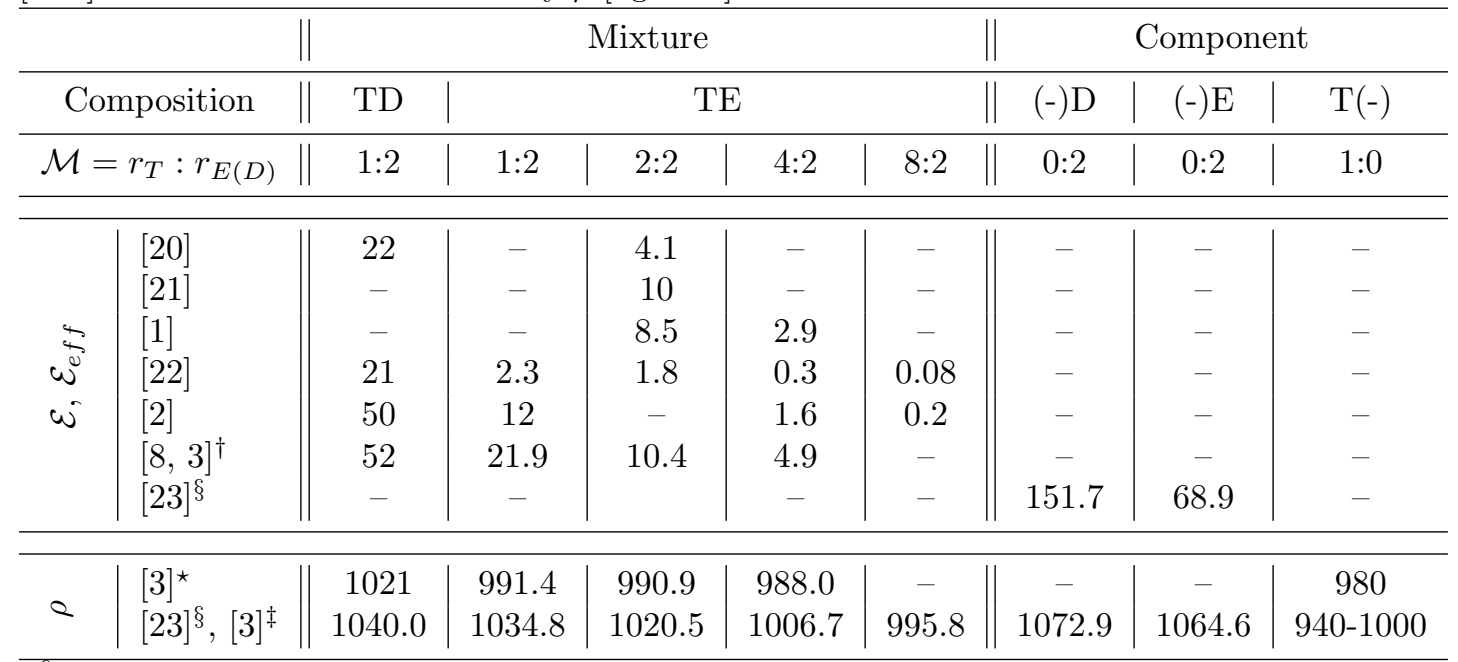

$\S$ from manufacturer data sheet for single components (-)D, (-)E and T(-)

${ }^{\dagger}$ values reported in Table 1 for the VF replicas shown in Fig. 2

* measured densities for the VF replicas shown in Fig. 2

$\ddagger$ computed mixture densities using Eq. (4) with $\rho_{T}=980 \mathrm{~kg} \cdot \mathrm{m}^{-3}$ 
the omitted component is replaced by (-). Properties for these individual components ((-)D, (-)E and $\mathrm{T}(-))$ are obtained from the manufacturer [23]. For silicone thinner $(\mathrm{T}(-))$, a density range is indicated so that its value $\rho_{T}$ needs to be measured [3]. The densities of TE and TD mixtures, $\rho_{T E}$ and $\rho_{T D}$, are both measured and computed from the mixing ratio $\mathcal{M}=r_{T}: r_{E(D)}$ as:

$$
\rho_{T E(D)}=\frac{r_{T}+r_{E(D)}}{\frac{r_{T}}{\rho_{T}}+\frac{r_{E(D)}}{\rho_{E(D)}}}
$$

where $\rho_{E}$ (or $\rho_{D}$ ) denotes the density of the component (-)E (or (-)D) provided by the manufacturer 23] and $\rho_{T}$ corresponds to the measured value $\left(\rho_{T}=980 \mathrm{~kg} \cdot \mathrm{m}^{-3}\right.$ in Table 2). Computed and measured mixture densities both increase with mass portion $r_{T}$ and their difference is less than $4.5 \%$ of measured mixture densities.

As the Young's modulus $\mathcal{E}$ of component $(-) \mathrm{D}$ is more than twice the value of component $(-) \mathrm{E}, \mathcal{E}_{\text {eff }}$ is larger for TD mixtures than for TE mixtures at similar mixing ratios $\left(r_{E}=r_{D}\right)$. Thus, TD mixtures are more rigid than similar TE mixtures. In addition, Young's modulus $\mathcal{E}_{\text {eff }}$ decreases as the mixing ratio $\mathcal{M}$, and hence the relative mass portion of silicone Thinner $r_{T}$, increases as explicitly observed for TE mixtures. Comparing literature values of $\mathcal{E}_{\text {eff }}$ at similar $\mathcal{M}$ reveals that their value and increase with $\mathcal{M}$ differs considerably between cited studies. This is illustrated for $\mathcal{E}_{\text {eff }}$ values reported for TE mixtures in [8, 3] and [2]: given $\mathcal{E}_{\text {eff }}$ values for $\mathcal{M}=1: 2$ differ with $45 \%$ and they decrease to respectively $22 \%$ [8, 3] or $13 \%$ [2] of their value for $\mathcal{M}=4: 2$ or a $67 \%$ difference. As molding mixture compositions in this work are prepared exactly (same components, procedure and equipment) as described in [3, 8], values herein are taken as a literature reference denoted $\mathcal{E}_{\text {eff }}^{r e f}$ hereafter.

\section{Analytical model of the effective linear Young's modulus}

155 ML composites are considered which consist of $n$ elastic, isotropic and perfectly bounded layers, which are themselves composed of one or more constituents. The linear stress-strain behaviour of a ML composite is then 


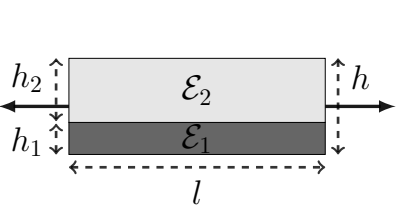

(a) parallel, $\|$

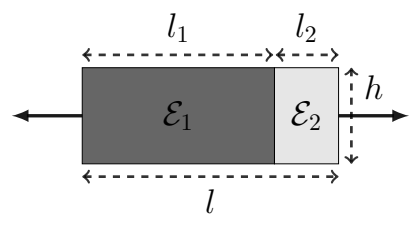

(b) serial, $\perp$

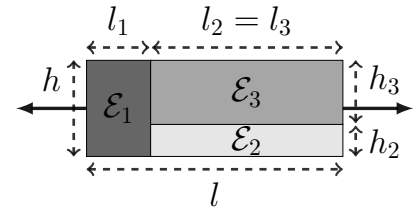

(c) combined, $\perp \|$

Figure 4: Layer stacking about the force direction (full arrows) in ML composites with the stacking orientation $o_{j, j+1} \in\{\perp, \|\}$ of adjacent layers: a) 2L parallel $(\|)$ with $o_{(1,2)}=\|$, b) $2 \mathrm{~L}$ serial $(\perp)$ with $o_{(1,2)}=\perp$, c) $3 \mathrm{~L}$ combined $(\perp \|)$ with $o_{(1,2)}=\perp$ and $o_{(2,3)}=\|$.

described in the same way as for each individual layer by attributing an effective Young's modulus $\mathcal{E}_{\text {eff }}$ describing the linear stress-strain behaviour for an equivalent homogeneous elastic composite. It follows that Eq. (3) holds for each layer and for the equivalent homogeneous ML composite. An analytical model is sought predicting $\mathcal{E}_{\text {eff }}$ for the equivalent homogeneous composite for which adjacent layers are stacked either parallel $(\|)$ or serial $(\perp)$ with respect to the force or stress direction. Stacking orientations between adjacent layers are denoted $o_{(j, j+1)} \in\{\perp, \|\}$ with $j=1 \ldots n-1$. The stacking orientation is assumed to remain similar during all deformation stages. Thus, besides parallel (Fig. 4(a) or serial (Fig. 4(b) stacked composites, both stacking orientations $(\|$ and $\perp)$ can be combined to describe more complex ML composites (combined, $\perp \|$ ) as illustrated in Fig. 4(c), Each layer $i=1 \ldots n$ is characterised by its length $l_{i}$ (parallel to the force direction), height $h_{i}$ (transverse to the force direction) and Young's modulus $\mathcal{E}_{i}$. The effective Young's modulus $\mathcal{E}_{\text {eff }}$ for parallel or serial stacked layers is then modelled applying the theory of linear elasticity (Eq. (3)) to each layer and to the equivalent homogeneous composite.

For $n$ parallel stacked layers the force is distributed over the layers $(\mathcal{F}=$ $\sum_{i=1}^{n} \mathcal{F}_{i}$ ) so that the strain $\varepsilon$ in the equivalent homogeneous composite and the strain $\varepsilon_{i=1 \ldots n}$ in each layer is constant, i.e. $\varepsilon_{i=1 \ldots n}=\varepsilon$. The effective Young's modulus of the equivalent homogeneous composite with transverse height $h=\sum_{i=1}^{n} h_{i}$ is then modelled using the Voigt hypothesis [24] of ho- 
mogeneous deformation as

$$
\mathcal{E}_{\text {eff }}^{\|}=\frac{\sum_{i=1}^{n} h_{i} \cdot \mathcal{E}_{i}}{\sum_{i=1}^{n} h_{i}}
$$

Thus, $\mathcal{E}_{\text {eff }}^{\|}$is computed as the weighted arithmetic mean (WAM) of the layers Young's moduli $\mathcal{E}_{i}$. The arithmetic mean is weighted with transverse layer heights $h_{i}$, which amounts to applying the rule of mixtures.

For $n$ serial stacked layers the stress $\sigma$ in the equivalent homogeneous composite and the stress $\sigma_{i=1 \ldots n}$ in each layer is constant, i.e. $\sigma_{i=1 \ldots n}=\sigma$. The effective Young's modulus of the equivalent homogeneous composite with parallel length $l=\sum_{i=1}^{n} l_{i}$ is then modelled using the Reuss hypothesis [25] of homogeneous stress as

$$
\mathcal{E}_{\text {eff }}^{\perp}=\frac{\sum_{i=1}^{n} l_{i}}{\sum_{i=1}^{n}\left(\frac{l_{i}}{\mathcal{E}_{i}}\right)} .
$$

Thus, $\mathcal{E}_{\text {eff }}^{\perp}$ is obtained as the harmonic mean of the layers Young's moduli $\mathcal{E}_{i}$ weighted with their parallel lengths $l_{i}$. It is noted that applying the rule of mixtures would results in the weighted arithmetic mean of $\mathcal{E}_{i}$ instead of the weighted harmonic mean (WHM) expressed in Eq. (6).

185 Both Eq. (5) and Eq. (6) result in $\mathcal{E}_{\text {eff }}^{\perp, \|}=\mathcal{E}_{1}$ when $n=1$. Eq. (5) and Eq. (6) do not account for the stacking order so that modelled $\mathcal{E}_{\text {eff }}^{\|}$and $\mathcal{E}_{\text {eff }}^{\perp}$ remain unaffected when layers are permuted or splitted as long as the overall composition and orientation of the stack is maintained. It is well established that WAM values are larger than WHM values. Therefore, layers with large $\mathcal{E}_{i}$ tend to mask layers with lower $Y_{i}$ for modelled $\mathcal{E}_{\text {eff }}^{\|}$(WAM in Eq. (5)) whereas this is less the case for modelled $\mathcal{E}_{\text {eff }}^{\perp}$ (WHM in Eq. (6)).

The equivalent Young's modulus $\mathcal{E}_{\text {eff }}^{\perp \|}$ of more complex ML composites with combined $(\perp \|)$ stacking, composed of both serial and parallel layers as illustrated in Fig. 4(c), is modelled using a two-step approach. Firstly, 


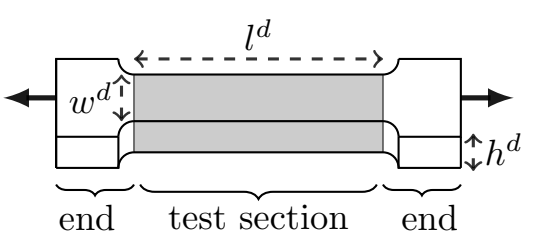

(a) design

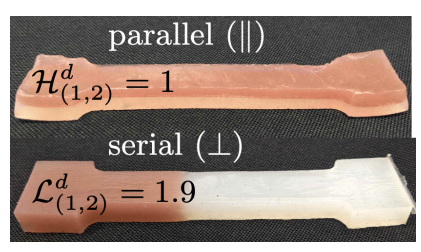

(b) 2L moldings

Figure 5: Bone-shaped specimens: a) design (superscript $d$ ) for uni-axial stress testing (black arrows), end terminations for clamping and the test section (shaded) with $l^{d}=$ $80 \mathrm{~mm}, h^{d}=10 \mathrm{~mm}$ and $\left.w^{d}=15 \mathrm{~mm}, \mathrm{~b}\right)$ molded parallel $(\|)$ and serial $(\perp) 2 \mathrm{~L}$ composites (colors) for stacking dimension ratios $\mathcal{H}_{(1,2)}^{d}=h_{1} / h_{2}$ and $\mathcal{L}_{(1,2)}^{d}=l_{1} / l_{2}$.

Eq. (5) is used to homogenise parallel stacked layers. Secondly, Eq. (6) is applied to the resulting stack of serial layers. As WAM (Eq. (5)) and WHM (Eq. (6)) value layers with large $\mathcal{E}_{i}$ differently, the layer order might affect the model value of $\mathcal{E}_{\text {eff }}^{\perp \|}$, which is not the case for ML composites consisting of serial or parallel layers only.

\section{Model analysis and selected specimen designs}

In this section the $\mathcal{E}_{\text {eff }}$ model approach outlined in Section 3 is analysed for bone-shaped ML composites with two or three layers stacked parallel (Eq. (5), WAM), serial (Eq. (6), WHM) or combined (Eq. (5) followed by Eq. (6), WAM followed by WHM). The model analysis is then used to select and motivate the designs of bone-shaped specimen suitable to validate the model approach outlined in section 3 against experimental values obtained from uni-axial stress testing outlined in section 6. Design values are indicated with superscript $d$. Model analysis is then assessed for bone-shaped specimen designs containing a test section with dimensions $l^{d}=80 \mathrm{~mm}$, $h^{d}=10 \mathrm{~mm}, w^{d}=15 \mathrm{~mm}$ and volume $\mathcal{V}^{d}=12 \mathrm{~cm}^{3}$ as depicted in Fig. 5(a). Layers are indicated with indexes $i=1 \ldots n$ and $j=1 \ldots n-1$. The layer width is held constant so that $w_{i}^{d}=w^{d}$ for all designs layers regardless of the stacking orientation. The geometrical specimen designs are then characterised from the height dimension ratio $\mathcal{H}^{d}$ and the length dimension ratio 
$\mathcal{L}^{d}$ between adjacent stacked layers:

$$
\begin{aligned}
\mathcal{H}_{(j, j+1)}^{d} & =\frac{h_{j}^{d}}{h_{j+1}^{d}}, \\
\mathcal{L}_{(j, j+1)}^{d} & =\frac{l_{j}^{d}}{l_{j+1}^{d}} .
\end{aligned}
$$

For two adjacent parallel stacked layers $\left(o_{(j, j+1)}=\|\right.$ and $\left.l_{j}^{d}=l_{j+1}^{d}\right)$, the layers geometry is characterised by the height stacking dimension ratio $\mathcal{H}_{(j, j+1)}^{d}$ since $\mathcal{L}_{(j, j+1)}^{d}=1$ is constant. Similarly, for two adjacent serial stacked layers $\left(o_{(j, j+1)}=\perp\right.$ and $\left.h_{j}^{d}=h_{j+1}^{d}\right)$, the layers geometry is characterised by the length stacking dimension ratio $\mathcal{L}_{(j, j+1)}^{d}$ as $\mathcal{H}_{(j, j+1)}^{d}=1$ is constant. Consequently, the stacking geometry of ML specimens is fully defined by the series of stacking dimension ratios $\nabla_{(j, j+1)}^{d} \in\left\{\mathcal{H}_{(j, j+1)}^{d}, \mathcal{L}_{(j, j+1)}^{d}\right\}$ associated with the stacking orientation between adjacent layers $o_{(j, j+1)}$. It follows that for ML composites composed solely with either parallel or serial stacked layers, the series reduces to $\nabla_{(j, j+1)}^{d}=\mathcal{H}_{(j, j+1)}^{d}$ or $\nabla_{(j, j+1)}^{d}=\mathcal{L}_{(j, j+1)}^{d}$, respectively. Moreover, the design of ML composite specimens must satisfy $0.1 \leq \nabla_{(j, j+1)}^{d} \leq 6.4$ in order to match the order of magnitude associated with the ratio of adjacent layer thicknesses for the silicone VF replicas outlined in section 2. The specimen designs composition is characterised from the composition ratio between the Young's moduli of adjacent layers as:

$$
\mathcal{B}_{(j, j+1)}^{d}=\frac{\left(\mathcal{E}_{\text {eff }}^{d}\right)_{j}}{\left(\mathcal{E}_{\text {eff }}^{d}\right)_{j+1}}
$$

Each layer must contain one of the mixtures pertinent to silicone VF replicas described in section 2. Therefore, model analysis of $\mathcal{E}_{\text {eff }}^{d}$ is assessed for $0.2 \leq \mathcal{B}_{(j, j+1)} \leq 5$ within the range pertinent for silicone VF replicas. In the following, first (Section 4.1) three single layer compositions are detailed resulting in three one-layer $(1 \mathrm{~L}, n=1)$ specimen designs, next (Section 4.2) model analysis motivates the designs of six two-layer $(2 \mathrm{~L}, n=2)$ specimen and finally (in Section 4.3) the designs of seven three-layer $(3 \mathrm{~L}, n=3$ ) specimens is justified.

\section{1. $1 L$ specimen design}

One-layer specimens, labelled I, are designed in order to address the reproducibility of TD and TE mixtures compared to reference values $\mathcal{E}_{\text {eff }}^{\text {ref }}$ 
Table 3: 1L specimen design: label, mixture, mixing ratio $\mathcal{M}$, reference $\mathcal{E}_{\text {eff }}^{r e f}$ [3, 8].

\begin{tabular}{c||c|c|c}
\hline Specimen & mixture & ratio $\mathcal{M}$ & $\mathcal{E}_{\text {eff }}^{\text {ref }}[\mathrm{kPa}]$ \\
\hline \hline $\mathrm{I}_{1}$ & TD & $1: 2$ & 52.0 \\
$\mathrm{I}_{2}$ & TE & $2: 2$ & 10.4 \\
$\mathrm{I}_{3}$ & TE & $4: 2$ & 4.9 \\
\hline
\end{tabular}

in [3, 8] as literature values (Table 2) of $\mathcal{E}_{\text {eff }}$ for similar moldings vary considerable, e.g. $67 \%$ for ES $\mathcal{M}=4: 2$ as $\mathcal{E}_{\text {eff }}^{\text {ref }}=4.9 \mathrm{kPa}$ in [3, 8] compared to $\mathcal{E}_{\text {eff }}=1.6 \mathrm{kPa}$ in [2]. Single layer design dimensions of a $1 \mathrm{~L}$ specimen match the dimensions of the test section, i.e. $l_{1}^{d}=l^{d}, h_{1}^{d}=h^{d}$ and $w_{1}^{d}=w^{d}$. An overview of $1 \mathrm{~L}$ specimen designs is given in Table 3. The three 1L specimens, and hence their layer mixtures, are selected for three reasons: 1) there frequent use in ML silicone replicas (Table 1, 2) known reference $\mathcal{E}_{\text {eff }}^{\text {ref }}$ from [3, 8] and 3) the resulting $\mathcal{E}_{\text {eff }}^{\text {ref }}$-range $\left(4.9 \leq \mathcal{E}_{\text {eff }}^{\text {ref }} \leq 52 \mathrm{kPa}\right)$ overlaps most of the $\mathcal{E}$-range associated with anatomical VF layers $(\mathcal{E} \leq 60 \mathrm{kPa}$, Table 1). Indeed, reported $\mathcal{E}_{\text {eff }}^{\text {ref }}$ in Table 3 represent values reported for the muscle $\left(\mathrm{I}_{2}\right)$, superficial $\left(\mathrm{I}_{3}\right)$ and epithelium $\left(\mathrm{I}_{1}\right)$ layer of a human VF in Table 1 .

These three mixtures are then used in the model analysis and resulting designs of the 2L and 3L composite specimens outlined hereafter. The layer mixtures (and associated $\mathcal{E}_{\text {eff }}^{d}=\mathcal{E}_{\text {eff }}^{\text {ref }}$ in Table 3 for these ML specimens are indicated referring to the corresponding $1 \mathrm{~L}$ specimen, i.e. as $\mathrm{I}_{1}$, as $\mathrm{I}_{2}$ or as $\mathrm{I}_{3}$.

\subsection{L specimen design}

Two-layer composite specimens, labelled II, consist of two parallel $\left(o_{(1,2)}=\|\right.$ in Fig. 4(a) or two serial $\left(o_{(1,2)}=\perp\right.$ in Fig. 4(b) stacked layers. The geometrical design is fully characterised considering the stacking dimension ratio between both layers given as $\nabla_{(1,2)}^{d}=\mathcal{H}_{(1,2)}^{d}$ for parallel $(\|)$ stacking and $\nabla_{(1,2)}^{d}=\mathcal{L}_{(1,2)}^{d}$ for serial $(\perp)$ stacking. This is illustrated for color-molded specimens in Fig. 5(b), An overview of the $2 \mathrm{~L}$ specimen designs and its characteristics is given in Table 4 .

Modelled curves for $\mathcal{E}_{\text {eff }}^{\|, d}$ (from Eq. (5)) and for $\mathcal{E}_{\text {eff }}^{\perp, d}$ (from Eq. (6)) as a function of their stacking dimension ratio are plotted in Fig. 6(a) and Fig. 6(b), respectively. Modelled values for three different 2L composition 
Table 4: 2L specimen design: label, stacking orientation $o_{(1,2)}$, layer composition, stacking composition ratio $\mathcal{B}_{(1,2)}^{\text {ref }}$, stacking dimension ratio $\mathcal{H}_{(1,2)}^{d}$ or $\mathcal{L}_{(1,2)}^{d}$, modelled $\mathcal{E}_{\text {eff }}^{, d}$.

\begin{tabular}{|c|c|c|c|c|c|c|}
\hline \multirow{2}{*}{ Specimen } & \multirow{2}{*}{$o_{(1,2)}$} & \multicolumn{2}{|c|}{ composition } & \multirow{2}{*}{$\mathcal{B}_{(1,2)}^{r e f}$} & \multirow{2}{*}{$\mathcal{H}_{(1,2)}^{d}, \mathcal{L}_{(1,2)}^{d}$} & \multirow{2}{*}{$\begin{array}{c}\text { model } \\
\mathcal{E}_{\text {eff }}^{,, d} \\
\end{array}$} \\
\hline & & layer 1 & layer 2 & & & \\
\hline $\mathrm{II}_{1, \|}$ & $\|$ & as $\mathrm{I}_{2}$ & as $I_{3}$ & 2.1 & $\mathcal{H}^{d}=5.0$ & $9.5 \mathrm{kPa}$ \\
\hline $\mathrm{II}_{2, \|}$ & $\|$ & as $\mathrm{I}_{2}$ & as $\mathrm{I}_{3}$ & 2.1 & $\mathcal{H}^{d}=1.0$ & $7.7 \mathrm{kPa}$ \\
\hline $\mathrm{II}_{3, \|}$ & $\|$ & as $\mathrm{I}_{1}$ & as $I_{2}$ & 5.0 & $\mathcal{H}^{d}=1.0$ & $31.2 \mathrm{kPa}$ \\
\hline $\mathrm{II}_{1, \perp}$ & $\perp$ & as $\mathrm{I}_{2}$ & as $\mathrm{I}_{3}$ & 2.1 & $\mathcal{L}^{d}=5.0$ & $8.8 \mathrm{kPa}$ \\
\hline $\mathrm{II}_{2, \perp}$ & $\perp$ & as $\mathrm{I}_{2}$ & as $I_{3}$ & 2.1 & $\mathcal{L}^{d}=1.0$ & $6.7 \mathrm{kPa}$ \\
\hline $\mathrm{II}_{3, \perp}$ & $\perp$ & as $I_{1}$ & as $\mathrm{I}_{2}$ & 5.0 & $\mathcal{L}^{d}=1.0$ & $17.3 \mathrm{kPa}$ \\
\hline
\end{tabular}

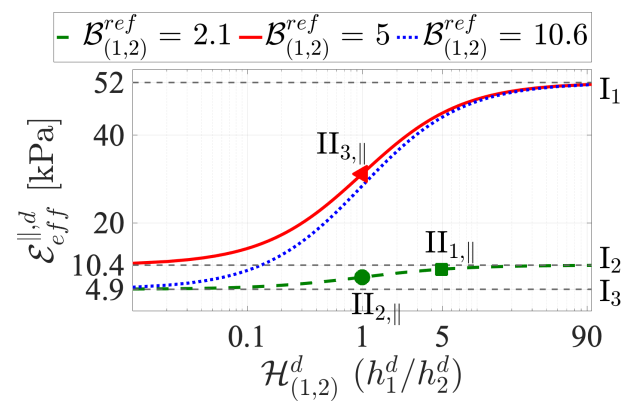

(a) $2 \mathrm{~L}$, parallel $(\|)$

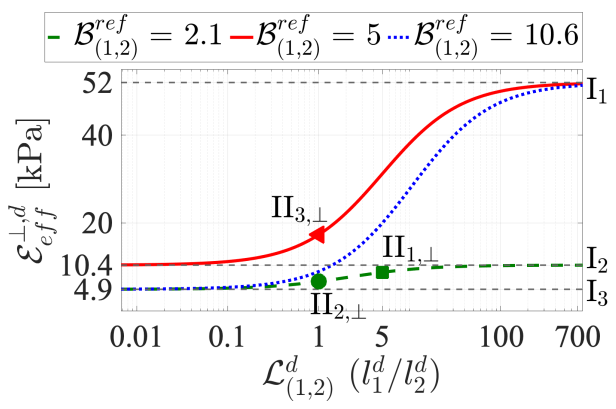

(b) $2 \mathrm{~L}$, serial $(\perp)$

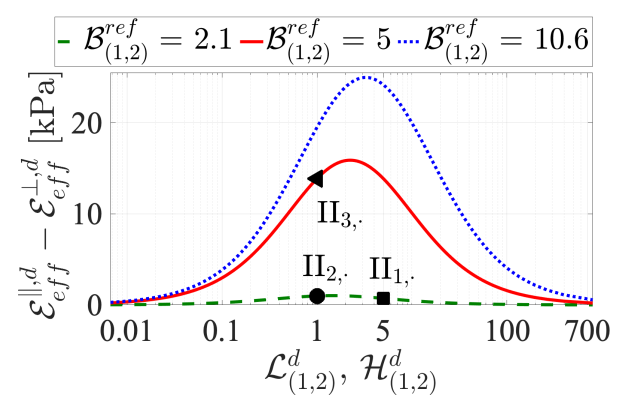

(c) 2L, WAM $(\|)$ minus WHM $(\perp)$

Figure 6: Modelled $\mathcal{E}_{\text {eff }}^{, d}$ for $2 \mathrm{~L}$ stacking for different $\mathcal{B}_{(1,2)}^{\text {ref }}$ as a function of dimension ratio. $2 \mathrm{~L}$ specimen design values (symbols) are annotated (II., or $\left.\mathrm{II}_{., \perp}\right)$ : a) $\mathcal{H}_{(1,2)}^{d}$ for parallel $(\|)$, b) $\mathcal{L}_{(1,2)}^{d}$ for serial $(\perp)$. Horizontal dashed lines indicate $\mathcal{E}_{\text {eff }}^{\text {ref }}$ of individual layers (I.), c) averaging (WAM (\|) or WHM $(\perp)$ ) induced difference $\mathcal{E}_{\text {eff }}^{\|, d}-\mathcal{E}_{\text {eff }}^{\perp, d}$. 
ratios $\mathcal{B}_{(1,2)}^{\text {ref }}$ are shown, i.e. $\mathcal{B}_{(1,2)}^{\text {ref }}=2.1$ for mixtures $\left(\right.$ as $\mathrm{I}_{2}$, as $\left.\mathrm{I}_{3}\right), \mathcal{B}_{(1,2)}^{\text {ref }}=5$ for mixtures (as $\mathrm{I}_{1}$, as $\mathrm{I}_{2}$ ) and $\mathcal{B}_{(1,2)}^{\text {ref }}=10.6$ for mixtures (as $\mathrm{I}_{1}$, as $\mathrm{I}_{3}$ ). As a reference, $\mathcal{E}_{\text {eff }}^{\text {ref }}$ for $1 \mathrm{~L}$ specimens are indicated (horizontal dashed lines annotated $\left.\mathrm{I}_{1,2,3}\right)$. The shown stacking dimension ratio ranges $\left(0<\mathcal{H}_{(1,2)}^{d} \leq 700\right.$ and $\left.0<\mathcal{L}_{(1,2)}^{d} \leq 90\right)$ are adapted so that modelled $\mathcal{E}_{\text {eff }}^{, d}$ vary in the range between single layer values $\left(\mathcal{E}_{\text {eff }}^{r e f}\right)_{i=2}$ and $\left(\mathcal{E}_{\text {eff }}^{\text {ref }}\right)_{i=1}$. For similar dimension ratios $\left(\mathcal{L}_{(1,2)}^{d}=\mathcal{H}_{(1,2)}^{d}\right)$ and composition ratios $\mathcal{B}_{(1,2)}^{\text {ref }}$, modelled $\mathcal{E}_{\text {eff }}^{\cdot d}$ values for serial and for parallel stacking differ only due to the applied averaging, i.e. arithmetic (WAM) for parallel stacking in Eq. (5) and harmonic (WHM) for serial stacking in Eq. (6). The resulting inter-model difference, $\mathcal{E}_{\text {eff }}^{\|, d}-\mathcal{E}_{\text {eff }}^{\perp, d} \geq 0$, due to the stacking orientation is plotted in Fig. 6(c). The curves exhibit a maximum for dimension ratios in the range of interest $\left(0.1 \leq \nabla_{(j, j+1)}^{d} \leq 6.4\right)$ and tend to zero for very small or very large ratios for which $\mathcal{E}_{\text {eff }}^{, d} \approx\left(\mathcal{E}_{\text {eff }}^{\text {ref }}\right)_{2}$ and $\mathcal{E}_{\text {eff }}^{\cdot, d} \approx\left(\mathcal{E}_{\text {eff }}^{r e f}\right)_{1}$, respectively. The difference increases with composition ratio $\mathcal{B}_{(1,2)}^{\text {ref }}$ reflecting the increasing impact of layers with large $\mathcal{E}_{\text {eff }}^{\text {ref }}$ to modelled $\mathcal{E}_{\text {eff }}^{\|, d}$ values. This is also seen from Fig. 6(a) and Fig. 6(b), e.g. comparing dimension ratios at which $\mathcal{E}_{\text {eff }}^{, d}$ increases from its lowest value $\left(\mathcal{E}_{\text {eff }}^{r e f}\right)_{i=2}$.

Modelled curves show that stacking dimension ratios $\nabla_{(1,2)}^{d}=\{1,5\}$ are suitable for $2 \mathrm{~L}$ specimen design as modelled $\mathcal{E}_{\text {eff }}^{, d}$ differ between both stacking orientations $o_{(1,2)} \in\{\|, \perp\}$, vary with dimension ratio $\nabla_{(j, j+1)}^{d}$ and differ from layer values $\left(\mathcal{E}_{\text {eff }}^{r e f}\right)_{i}$ for all $\mathcal{B}_{(1,2)}^{\text {ref }}$. Model curves for $\mathcal{B}_{(1,2)}^{\text {ref }} \in\{2.1,5.0\}$ (dashed and full curve in Fig. 60 enclose the curve for $\mathcal{B}_{(1,2)}^{\text {ref }}=10.6$ (dotted curve in Fig. 6) so that the influence of the stacking composition on $\mathcal{E}_{\text {eff }}^{d}$ is larger considering $\mathcal{B}_{(1,2)}^{\text {ref }} \in\{2.1,5.0\}$ for $2 \mathrm{~L}$ specimen design. The $2 \mathrm{~L}$ specimens are thus designed so that for each stacking orientation $o_{(1,2)} \in\{\|, \perp\}$ the influence of stacking composition $\mathcal{B}_{(1,2)}^{\text {ref }} \in\{2.1,5.0\}\left(\mathrm{II}_{2, \text {. versus }} \mathrm{II}_{3, .}\right)$ and stacking dimension ratio $\nabla_{(1,2)}^{d} \in\{1,5\}\left(\mathrm{II}_{1,}\right.$. versus $\left.\mathrm{II}_{2, .}\right)$ on the modelled $\mathcal{E}_{\text {eff }}^{\cdot, d}$ can be evaluated. The influence of stacking orientation $o_{(1,2)}$ on modelled $\mathcal{E}_{\text {eff }}^{\cdot, d}$ can be assessed as well (II., ${ }^{\prime}$ versus II., $)$. Modelled values $\mathcal{E}_{\text {eff }}^{\cdot, d}$ for $2 \mathrm{~L}$ specimen designs are reported in Table 4 and indicated (symbols annotated with the specimen label II.,.) in Fig. 6. 
Table 5: 3L specimen design: label, stacking orientation $o$, layer composition, stacking composition ratios $\mathcal{B}^{\text {ref }}$, stacking dimension ratio $\nabla^{d} \in\left\{\mathcal{H}^{d}, \mathcal{L}^{d}\right\}$, modelled $\mathcal{E}_{\text {eff }}^{\cdot, d}$.

\begin{tabular}{|c|c|c|c|c|c|c|c|c|c|c|}
\hline \multirow[t]{2}{*}{ Specimen } & \multicolumn{2}{|c|}{$o_{(j, j+1)}$} & \multicolumn{3}{|c|}{ layer $i$ composition } & \multicolumn{2}{|c|}{$\mathcal{B}_{(j, j+1)}^{r e f}$} & \multicolumn{2}{|c|}{$\mathcal{H}_{(j, j+1)}^{d}, \mathcal{L}_{(j, j+1)}^{d}$} & \multirow{2}{*}{$\begin{array}{c}\text { model } \\
\mathcal{E}_{\text {eff }}^{\cdot, d}\end{array}$} \\
\hline & $(1,2)$ & $(2,3)$ & 1 & 2 & 3 & $(1,2)$ & $(2,3)$ & $(1,2)$ & $(2,3)$ & \\
\hline $\begin{array}{l}\mathrm{III}_{1, \perp} \\
\mathrm{III}_{2, \perp}\end{array}$ & $\begin{array}{l}\perp \\
\perp\end{array}$ & $\begin{array}{l}\perp \\
\perp\end{array}$ & $\begin{array}{l}\text { as } \mathrm{I}_{2} \\
\text { as } \mathrm{I}_{1}\end{array}$ & $\begin{array}{l}\text { as } \mathrm{I}_{3} \\
\text { as } \mathrm{I}_{2}\end{array}$ & $\begin{array}{l}\text { as } \mathrm{I}_{2} \\
\text { as } \mathrm{I}_{1}\end{array}$ & $\begin{array}{l}2.1 \\
5.0\end{array}$ & $\begin{array}{c}0.47 \\
0.2\end{array}$ & $\begin{aligned} \mathcal{L}^{d} & =0.50 \\
\mathcal{L}^{d} & =0.50\end{aligned}$ & $\begin{aligned} \mathcal{L}^{d} & =2.0 \\
\mathcal{L}^{d} & =2.0\end{aligned}$ & $\begin{array}{r}6.7 \mathrm{kPa} \\
17.3 \mathrm{kPa}\end{array}$ \\
\hline $\begin{array}{l}\mathrm{III}_{3, \perp} \\
\mathrm{III}_{4, \perp} \\
\mathrm{III}_{1, \|} \\
\mathrm{III}_{1, \perp \|} \\
\mathrm{III}_{2, \perp \|}\end{array}$ & $\begin{array}{l}\perp \\
\perp \\
\| \\
\perp \\
\perp\end{array}$ & $\begin{array}{l}\perp \\
\perp \\
\| \\
\| \\
\|\end{array}$ & as $\mathrm{I}_{1}$ & as $\mathrm{I}_{2}$ & as $\mathrm{I}_{3}$ & 5.0 & 2.1 & $\begin{array}{c}\mathcal{L}^{d}=0.66 \\
\mathcal{L}^{d}=4.2 \\
\mathcal{H}^{d}=0.50 \\
\mathcal{L}^{d}=0.14 \\
\mathcal{L}^{d}=2.1\end{array}$ & $\begin{aligned} \mathcal{L}^{d} & =1.0 \\
\mathcal{L}^{d} & =1.0 \\
\mathcal{H}^{d} & =1.0 \\
\mathcal{H}^{d} & =1.5 \\
\mathcal{H}^{d} & =1.5\end{aligned}$ & $\begin{array}{r}8.5 \mathrm{kPa} \\
16.2 \mathrm{kPa} \\
16.5 \mathrm{kPa} \\
8.7 \mathrm{kPa} \\
14.4 \mathrm{kPa}\end{array}$ \\
\hline
\end{tabular}

\subsection{L specimen design}

Three-layer composite specimens, labelled III, consist of three layers with 2 or 3 different mixtures. Adjacent layers are stacked either parallel or serial so that $o_{(1,2)}, O_{(2,3)} \in\{\|, \perp\}$. Dimension ratios are chosen within the range of interest $0.1 \leq \nabla_{(1,2)}^{d}, \nabla_{(2,3)}^{d} \leq 6.4$. The $3 \mathrm{~L}$ composition is characterised by composition ratios $\mathcal{B}_{(1,2)}^{\text {ref }}$ and $\mathcal{B}_{(2,3)}^{\text {ref }}$. An overview of the $3 \mathrm{~L}$ specimen designs is given in Table 5 .

Two 3L composite specimens $\left(\mathrm{III}_{1, \perp}\right.$ and $\left.\mathrm{III}_{2, \perp}\right)$ with serial stacking $\left(o_{(j, j+1)}=\perp\right)$ are designed in order to evaluate the model property that layer splitting and layer permutation do not affect modelled $\mathcal{E}_{\text {eff }}^{\perp, d}$. These specimens have the same overall composition as $2 \mathrm{~L}$ specimens $\mathrm{II}_{2, \perp}$ and $\mathrm{II}_{3, \perp}$ respectively, so that modelled $\mathcal{E}_{\text {eff }}^{\perp, d} \in\{6.7,17.3\} \mathrm{kPa}$ in Table 5 and Table 4 match. Both 3L specimens are obtained by permuting half of the first layer $(i=1)$ of the $2 \mathrm{~L}$ specimens to form a third layer $(i=3)$ on top of the second layer $(i=2)$ so that the dimension ratio $\mathcal{L}_{1,2}^{d}=0.5$ of the resulting $3 \mathrm{~L}$ specimens amounts to half of the $2 \mathrm{~L}$ specimens value and $\mathcal{L}_{2,3}^{d}=\left(\mathcal{L}_{1,2}^{d}\right)^{-1}$. It follows that $\left(\mathcal{E}_{\text {eff }}^{\text {ref }}\right)_{1}=\left(\mathcal{E}_{\text {eff }}^{\text {ref }}\right)_{3}$ so that $\mathcal{B}_{(1,2)}^{\text {ref }}=\left(\mathcal{B}_{(2,3)}^{\text {ref }}\right)^{-1}$ with $\mathcal{B}_{(1,2)}^{\text {ref }} \in\{2.1,5\}$ as for the $2 \mathrm{~L}$ specimens in Table 4. Serial $(\perp$, WHM in Eq. (6) $)$ and not parallel (\|, WAM in Eq. (5)) stacked specimens are considered as modelled $\mathcal{E}_{\text {eff }}^{\perp, d}$ are less affected by layers with large $\mathcal{E}_{\text {eff }}^{r e f}$ than modelled $\mathcal{E}_{\text {eff }}^{\|, d}$, so that the potential influence of layer permutation in a parallel stack is more likely to go unnoticed. 


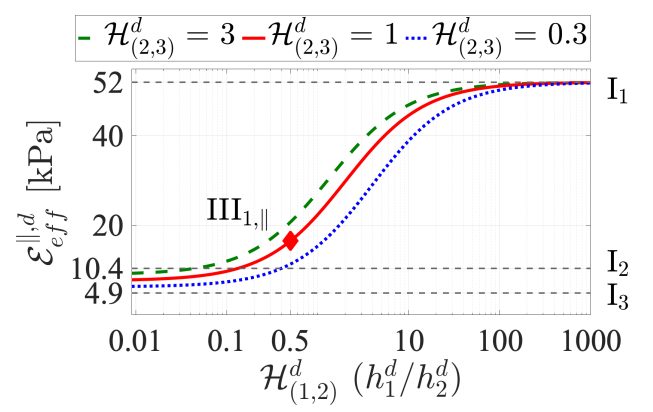

(a) $3 \mathrm{~L}$, parallel $(\|)$

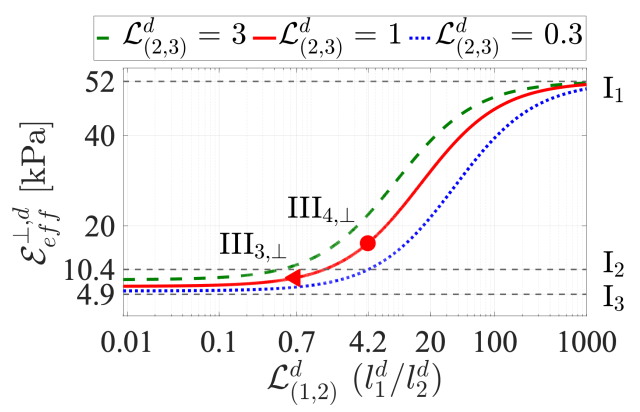

(b) $3 \mathrm{~L}$, serial $(\perp)$

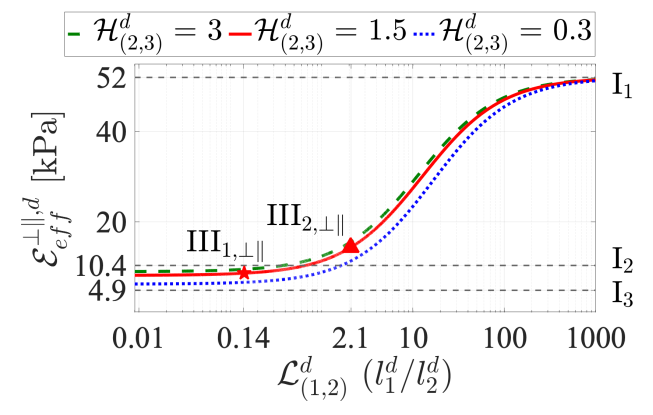

(c) $3 \mathrm{~L}$, combined $(\perp \|)$

Figure 7: Modelled $\mathcal{E}_{\text {eff }}^{, d}$ for $3 \mathrm{~L}$ stacking with $\mathcal{B}_{(1,2)}^{\text {ref }}=5$ and $\mathcal{B}_{(2,3)}^{\text {ref }}=2.1$ as a function of dimension ratio $\nabla_{(1,2)}^{d}$ for three different dimension ratios $\nabla_{(2,3)}^{d}$. 3L specimen design values (symbols) are annotated (III.,, III.,, or III.,,$\|)$ : a) parallel, $o_{(j, j+1)}=\|$ and $\nabla_{(j, j+1)}^{d}=\mathcal{H}_{(j, j+1)}^{d}$, b) serial, $o_{(j, j+1)}=\perp$ and $\nabla_{(j, j+1)}^{d}=\mathcal{L}_{(j, j+1)}^{d}$ and c) combined $o_{(1,2)}=\perp$ and $o_{(2,3)}=\|$ so that $\nabla_{(1,2)}^{d}=\mathcal{L}_{(1,2)}^{d}$ and $\nabla_{(2,3)}^{d}=\mathcal{H}_{(2,3)}^{d}$. Horizontal dashed lines indicate $\mathcal{E}_{\text {eff }}^{\text {ref }}$ of individual layers (I.). 
Different stacking orientations $\left(o_{(1,2)}, o_{(2,3)} \in\{\|, \perp\}\right)$ and dimension ratios $\left(\nabla_{(1,2)}^{d}\right.$ and $\left.\nabla_{(2,3)}^{d}\right)$ are considered for the design of five 3L composite specimens $\left(\mathrm{III}_{3, \perp}, \mathrm{III}_{4, \perp}, \mathrm{III}_{1, \|}, \mathrm{III}_{1, \perp \|}\right.$ and $\left.\mathrm{III}_{2, \perp \|}\right)$ with three different layer mixtures. The same mixtures (as $\mathrm{I}_{1}$ in layer $i=1$, as $\mathrm{I}_{2}$ in layer $i=2$, as $\mathrm{I}_{3}$ in layer $i=3$ ) are considered, so that $\left(\mathcal{E}_{\text {eff }}^{\text {ref }}\right)_{i=1}>\left(\mathcal{E}_{\text {eff }}^{\text {ref }}\right)_{i=2}>\left(\mathcal{E}_{\text {eff }}^{\text {ref }}\right)_{i=3}$ result in constant composition ratios $\mathcal{B}_{(1,2)}^{\text {ref }}=5$ from mixtures (as $\mathrm{I}_{1}$, as $\mathrm{I}_{2}$ ) and $\mathcal{B}_{(2,3)}^{\text {ref }}=2.1$ from mixtures $\left(\right.$ as $\mathrm{I}_{2}$, as $\mathrm{I}_{3}$ ). The specimens are stacked either parallel $\left(o_{(1,2)}, o_{(2,3)}=\|, \nabla_{(j, j+1)}^{d}=\mathcal{H}_{(j, j+1)}^{d}\right)$, serial $\left(o_{(1,2)}, o_{(2,3)}=\perp\right.$, $\left.\nabla_{(j, j+1)}^{d}=\mathcal{L}_{(j, j+1)}^{d}\right)$ or combined $\left(o_{(1,2)}=\perp, o_{(2,3)}=\|, \nabla_{(1,2)}^{d}=\mathcal{L}_{(1,2)}^{d}, \nabla_{(2,3)}^{d}=\right.$ $\left.\mathcal{H}_{(2,3)}^{d}\right)$ as schematically illustrated in Fig. 4(c).

Modelled curves for $\mathcal{E}_{\text {eff }}^{\|, d}$ (from Eq. (5)), $\mathcal{E}_{\text {eff }}^{\perp, d}$ (from Eq. (6)) and $\mathcal{E}_{\text {eff }}^{\| \perp, d}$ (from Eq. (5) followed by Eq. (6)) as a function of dimension ratio $\nabla_{(1,2)}^{d}$ are plotted in Fig. 7(a), Fig. 7(b) and Fig. 7(c), respectively. Modelled values for three different dimension ratios $0.3 \leq \nabla_{(2,3)}^{d} \leq 3$ are shown. As a reference, $\mathcal{E}_{\text {eff }}^{r e f}$ for $1 \mathrm{~L}$ specimens are indicated (horizontal dashed lines annotated $\left.\mathrm{I}_{1,2,3}\right)$. The shown stacking dimension ratio range $\left(0<\nabla_{(1,2)}^{d} \leq 1000\right)$ is adapted so that modelled $\mathcal{E}_{e f f}^{, d}$ vary within the range spanned between the smallest $\left(\mathcal{E}_{\text {eff }}^{r e f}\right)_{i=3}$ and largest $\left(\mathcal{E}_{\text {eff }}^{r e f}\right)_{i=1}$ single layer values. For large dimension ratios $\nabla_{1,2}^{d}$ modelled $\mathcal{E}_{\text {eff }}^{, d}$ approximate the largest single layer value ${ }_{320}\left(\mathcal{E}_{\text {eff }}^{r e f}\right)_{i=1}$ so that $\mathcal{E}_{\text {eff }}^{\cdot, d} \approx\left(\mathcal{E}_{\text {eff }}^{r e f}\right)_{i=1}$. However, for small dimension ratios $\nabla_{1,2}^{d}$, the $3 \mathrm{~L}$ specimen behaves as a $2 \mathrm{~L}$ specimen composed of layers $i=2$ and $i=3$ so that modelled values depend on $\nabla_{(2,3)}^{d}$ (and implied orientation $\left.o_{(2,3)}\right)$. Concretely, modelled values for small $\nabla_{1,2}^{d}$ vary in the range $\left(\mathcal{E}_{\text {eff }}^{r e f}\right)_{i=3}<\mathcal{E}_{\text {eff }}^{\cdot, d}<\left(\mathcal{E}_{\text {eff }}^{r e f}\right)_{i=2}$ as $\mathcal{E}_{\text {eff }}^{\cdot, d}$ increases towards $\left(\mathcal{E}_{\text {eff }}^{r e f}\right)_{2}$ with $\nabla_{(2,3)}^{d}$.

The layer stacking orientation influences the weighted average accounted for in the model for parallel $(\|$, WAM), serial $(\perp, \mathrm{WHM})$ or combined $(\perp \|$, WAM followed by WHM) stacked 3L specimen designs. The influence of stacking orientations on modelled values is evaluated considering inter-model differences for similar dimension ratios (and composition ratios as these are held constant) so that inter-model discrepancies are solely due to the applied averaging: $\mathcal{E}_{\text {eff }}^{\|, d}-\mathcal{E}_{\text {eff }}^{\perp, d}>0$ in Fig. $8(\mathrm{a})$, $\mathcal{E}_{\text {eff }}^{\|, d}-\mathcal{E}_{\text {eff }}^{\perp \|, d}>0$ in Fig. $8(\mathrm{~b})$ and 


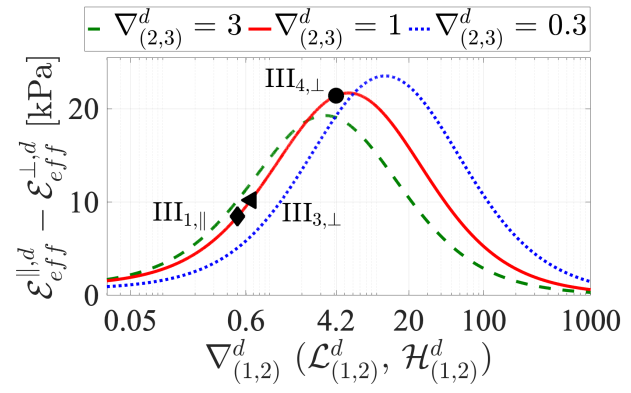

(a) $3 \mathrm{~L}, \|$ versus $\perp$

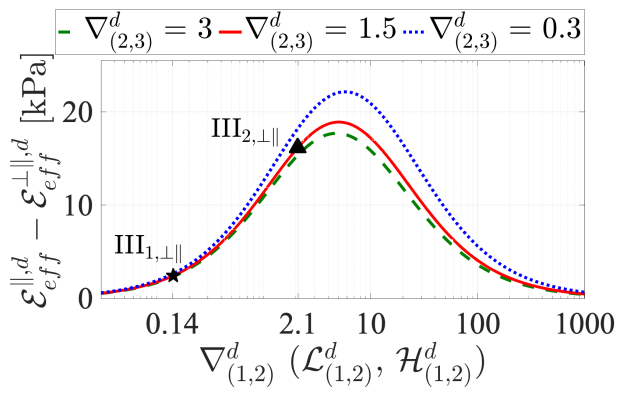

(b) $3 \mathrm{~L}, \|$ versus $\perp \|$

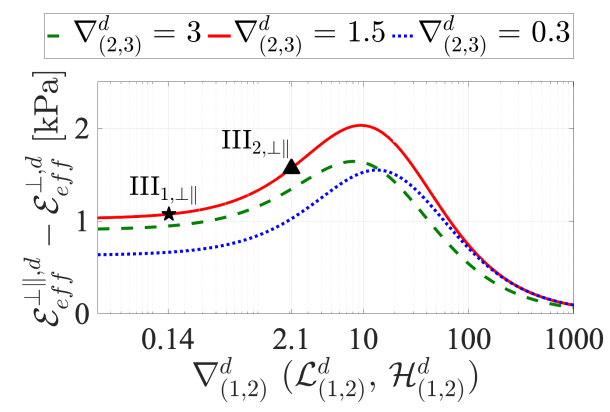

(c) $3 \mathrm{~L}, \perp \|$ versus $\perp$

Figure 8: Differences in modelled $\mathcal{E}_{\text {eff }}^{, d}$ due to 3 L layer stacking (parallel $(\|)$, serial $(\perp)$ or combined $(\perp \|))$ with $\mathcal{B}_{(1,2)}^{\text {ref }}=5$ and $\mathcal{B}_{(1,2)}^{\text {ref }}=2.1$ as a function of dimension ratio $\nabla_{(1,2)}^{d}$ for three different dimension ratios $\nabla_{(2,3)}^{d}$. 3L specimen design values (symbols) are annotated $\left(\mathrm{III}_{\cdot, \|}, \mathrm{III}_{,, \perp}\right.$ or III., $\left.\perp \|\right)$ : a) $\mathcal{E}_{\text {eff }}^{\|, d}-\mathcal{E}_{\text {eff }}^{\perp, d}$, b) $\mathcal{E}_{\text {eff }}^{\|, d}-\mathcal{E}_{\text {eff }}^{\perp \|, d}$, c) $\mathcal{E}_{\text {eff }}^{\perp \|, d}-\mathcal{E}_{\text {eff }}^{\perp, d}$. 
$\mathcal{E}_{\text {eff }}^{\perp \|, d}-\mathcal{E}_{\text {eff }}^{\perp, d}>0$ in Fig. $8(\mathrm{c})$. All plotted curves exhibit a maximum for dimension ratios $0.1<\nabla_{(1,2)}^{d}<11$ which is within or near the range of interest ${ }_{335}\left(0.1<\nabla_{(1,2)}^{d} \leq 6.4\right)$. As for small dimension ratios $\nabla_{(1,2)}^{d}$ each 3L specimen conducts itself as a $2 \mathrm{~L}$ specimen characterised by $\nabla_{(2,3)}^{d}$, plotted inter-model differences in this range are governed by $\nabla_{(2,3)}^{d}$. Therefore, 3L specimens with combined stacking $(\perp \|)$ perform as $2 \mathrm{~L}$ specimens with parallel stacking $(\|)$ so that in this range: 1) inter-model differences obtained comparing either "\| versus $\perp$ " or " $(\perp) \|$ versus $\perp$ " are similar (so for small $\nabla_{(1,2)}^{d}$ Fig. 8(c) zooms in on Fig. 8(a) and 2) inter-model comparison " $\|$ versus $(\perp) \|$ " (Fig. 8(b)) reduces to comparing $\|$ with itself yielding negligible inter-model differences regardless of $\nabla_{(2,3)}^{d}$. For very large $\nabla_{(1,2)}^{d}$ the influence of stacking orientation is small as for all stacking conditions $\mathcal{E}_{\text {eff }}^{\cdot, d}$ approximates single layer value $\mathrm{I}_{1}$. Within the range of interest $0.1<\nabla_{(1,2)}^{d}<6.4$, inter-model differences mostly increase with $\nabla_{(1,2)}^{d}$. Inter-model differences between serial $(\perp)$ and combined $(\perp \|)$ stacking (Fig. 8(c) remain limited to less than $2 \mathrm{kPa}$ whereas inter-model comparisons involving parallel (\|) stacking (Fig. 8(a) and Fig. $8(\mathrm{~b})$ amounts to larger (by a factor $\approx 10$ ) inter-model differences up to $23 \mathrm{kPa}$. This illustrates again the impact of a layer with large $\mathcal{E}_{\text {eff }}^{r e f}$, such as layer $i=1$ (by a factor 5 or more), when it is accounted for using WAM averages associated with parallel stacking $\left(o_{(1,2)}=\|\right)$ instead of WHM averages associated with serial or combined stacking $\left(o_{(1,2)}=\perp\right)$.

Curves in Fig. 7 and in Fig. 8 show that dimension ratios $\nabla_{(2,3)}^{d}$ near unity $\left(\nabla_{(2,3)}^{d} \in\{1,1.5\}\right)$ are suitable for 3L specimen designs. These ratios are then associated with both smaller $\left(0.1<\nabla_{(1,2)}^{d}<1\right)$ and larger $\left(1<\nabla_{(1,2)}^{d}<6.4\right)$ design dimension ratios $\nabla_{(1,2)}^{d}$ since modelled $\mathcal{E}_{e f f}^{, d}$ curves for larger $\nabla_{(1,2)}^{d}$ are more sensitive to the stacking orientation combination ( $\|, \perp$ or $\perp \|)$ and to the exact value of $\nabla_{(1,2)}^{d}$. The $3 \mathrm{~L}$ specimens (Table 5) with constant composition ratios $\mathcal{B}^{\text {ref }} \in\{5,2.1\}$ are thus designed with the aim of assessing the influence of geometrical design parameters as the stacking orientation on modelled $\mathcal{E}_{\text {eff }}^{, d}$ ( $" \mathrm{III}_{1, \|}$ versus $\mathrm{III}_{3, \perp}$ " and " $\mathrm{III}_{1, \|}$ versus $\mathrm{III}_{1, \perp \|}$ ") and the influence of $\nabla_{(1,2)}^{d}$ for different stacking combinations (" $\mathrm{III}_{3, \perp}$ versus $\mathrm{III}_{4, \perp}$ " and " $\mathrm{III}_{1, \perp \|}$ versus $\mathrm{III}_{2, \perp \|}$ "). 


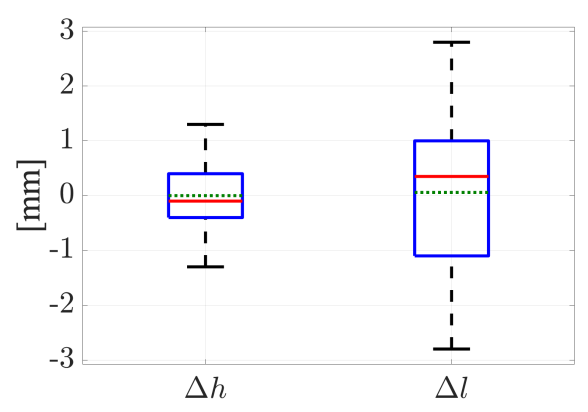

(a) $\Delta h, \Delta l[\mathrm{~mm}]$

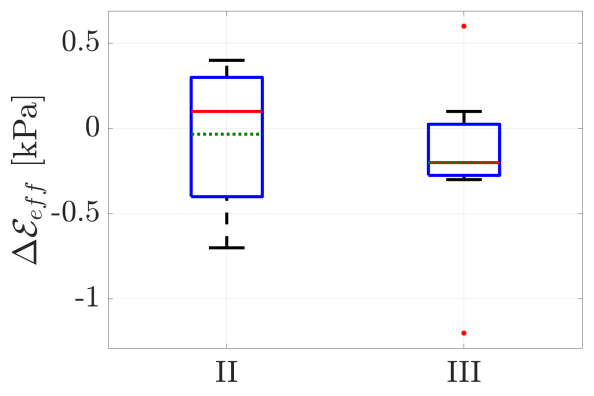

(b) $\Delta \mathcal{E}_{\text {eff }}[\mathrm{kPa}]$

Figure 9: Boxplots with median (full line), mean (dotted line), interquartile range between the first and third quartile (box), extrema (whiskers) of molding accuracies (molded minus design values) of: a) parallel $(\Delta h)$ and serial $(\Delta l)$ stacked layer dimensions, b) modelled $\Delta \mathcal{E}_{\text {eff }}$ for $2 \mathrm{~L}$ (II) and 3L (III) specimens.

\section{From designed to molded ML specimen: $\mathcal{E}_{\text {eff }}^{d}$ versus $\mathcal{E}_{\text {eff }}^{s-r e f}$}

Designed specimens are molded with a bone-shaped horizontal or vertical mold (volume $23.7 \mathrm{~cm}^{3}$ and 3D printed, Stratasys ABS-P430, accuracy $0.33 \mathrm{~mm}$ ) for parallel $(\|)$ and serial $(\perp)$ stacked layers respectively, following the mixture procedure outlined in [3, 8. All together, selected ML specimen designs contain 13 layers with parallel orientation and 24 layers with serial orientation. As specimens are molded layer by layer, the thickness of each molded layer along the molding direction is measured with a laser transceiver (Panasonic HL-G112-A-C5, wavelength $655 \mathrm{~nm}$, accuracy $8 \mu \mathrm{m}$ ). Measured layer dimensions are indicated with superscript $s$. The dimensional accuracy of each molded layer is obtained as the difference between the measured and designed dimension denoted $\Delta h$ and $\Delta l$ for a parallel and serial layer orientation, respectively. The repartition of dimensional molding accuracies $-2.8 \mathrm{~mm} \leq \Delta h, \Delta l \leq 2.8 \mathrm{~mm}$ is represented by a boxplot in Fig. 9(a) Overall, accuracies are characterised by their mean plus minus their standard deviation as $\Delta h=0.00 \pm 0.65 \mathrm{~mm}$ and $\Delta l=0.12 \pm 1.46 \mathrm{~mm}$, so that $\Delta h$ and $\Delta l$ are distributed around a small mean value near $0 \mathrm{~mm}$. The overall dimensional molding accuracy from both $\Delta h$ and $\Delta l$ yields $\pm 1.5 \mathrm{~mm}$.

Dimension ratios $\mathcal{H}^{s}$ and $\mathcal{L}^{s}$ of molded specimens (superscript $s$ ) are given in Table 6. Modelled $\mathcal{E}_{\text {eff }}^{s-r e f}$ values of molded specimens are obtained using 
Table 6: Molded 2L and 3L specimens: stacking dimension ratio $\nabla^{s} \in\left\{\mathcal{H}^{s}, \mathcal{L}^{s}\right\}$, modelled $\mathcal{E}_{\text {eff }}^{, s-r e f}$, relative model discrepancy $\xi_{\mathcal{E}_{\text {eff }}}$ between $\mathcal{E}_{\text {eff }}^{s-r e f}$ and $\mathcal{E}_{\text {eff }}^{d}$ associated with molded and designed specimen dimensions, respectively.

\begin{tabular}{|c|c|c|c|c|c|c|c|c|}
\hline \multicolumn{4}{|c|}{$2 \mathrm{~L}$ specimen } & \multicolumn{5}{|c|}{ 3L specimen } \\
\hline \multirow{2}{*}{ Label } & $\mathcal{H}^{s}, \mathcal{L}^{s}$ & \multicolumn{2}{|c|}{ model } & \multirow{2}{*}{ Label } & \multicolumn{2}{|c|}{$\mathcal{H}_{(j, j+1)}^{s}, \mathcal{L}_{(j, j+1)}^{s}$} & \multicolumn{2}{|c|}{ model } \\
\hline & $(1,2)$ & $\mathcal{E}_{e f f}^{\|, \perp, s-r e f}$ & $\xi_{\mathcal{E}_{\text {eff }}}$ & & $(1,2)$ & $(2,3)$ & $\mathcal{E}_{e f f}^{\cdot s-r e f}$ & $\xi_{\mathcal{E}_{e f f}}$ \\
\hline $\mathrm{II}_{1, \|}$ & $\mathcal{H}^{s}=2.3$ & $8.8 \mathrm{kPa}$ & $-7.4 \%$ & $\mathrm{III}_{1}$ & $\mathcal{L}^{s}=0.56$ & $\mathcal{L}^{s}=1.8$ & $6.8 \mathrm{kPa}$ & $1.5 \%$ \\
\hline $\mathrm{II}_{2, \|}$ & $\mathcal{H}^{s}=1.2$ & $7.9 \mathrm{kPa}$ & $2.6 \%$ & $\mathrm{III}_{2, \perp}$ & $\mathcal{L}^{s}=0.45$ & $\mathcal{L}^{s}=2.0$ & $17.1 \mathrm{kPa}$ & $-1.2 \%$ \\
\hline $\mathrm{II}_{3, \|}$ & $\mathcal{H}^{s}=1.0$ & $31.6 \mathrm{kPa}$ & $1.3 \%$ & $\mathrm{III}_{3, \perp}$ & $\mathcal{L}^{s}=0.68$ & $\mathcal{L}^{s}=0.91$ & $8.3 \mathrm{kPa}$ & $-2.4 \%$ \\
\hline $\mathrm{II}_{1, \perp}$ & $\mathcal{L}^{s}=6.6$ & $9.1 \mathrm{kPa}$ & $3.4 \%$ & $\mathrm{III}_{4, \perp}$ & $\mathcal{L}^{s}=4.2$ & $\mathcal{L}^{s}=1.1$ & $16.8 \mathrm{kPa}$ & $3.7 \%$ \\
\hline $\mathrm{II}_{2, \perp}$ & $\mathcal{L}^{s}=1.0$ & $6.7 \mathrm{kPa}$ & $0.0 \%$ & $\mathrm{III}_{1, \|}$ & $\mathcal{H}^{s}=0.51$ & $\mathcal{H}^{s}=0.74$ & $15.3 \mathrm{kPa}$ & $-7.3 \%$ \\
\hline \multirow[t]{2}{*}{$\mathrm{II}_{3, \perp}$} & $\mathcal{L}^{s}=0.93$ & $16.9 \mathrm{kPa}$ & $-2.3 \%$ & $\mathrm{III}_{1, \perp \|}$ & $\mathcal{L}^{s}=0.11$ & $\mathcal{H}^{s}=1.3$ & $8.4 \mathrm{kPa}$ & $-3.4 \%$ \\
\hline & & & & $\mathrm{III}_{2, \perp \|}$ & $\mathcal{L}^{s}=2.1$ & $\mathcal{H}^{s}=1.3$ & $14.2 \mathrm{kPa}$ & $-1.4 \%$ \\
\hline
\end{tabular}

for each layer the measured specimen dimensions and reference values $\mathcal{E}_{\text {eff }}^{\text {ref }}$ given in Table 3 . The relative molding accuracy $\xi_{\mathcal{E}_{\text {eff }}}$ for $2 \mathrm{~L}$ and $3 \mathrm{~L}$ specimens expresses then the relative model discrepancy of the Young's modulus due to layer molding dimension accuracy as $\xi_{\mathcal{E}_{\text {eff }}}=\Delta \mathcal{E}_{\text {eff }} / \mathcal{E}_{\text {eff }}^{d}$ with molding accuracy $\Delta \mathcal{E}_{\text {eff }}=\mathcal{E}_{\text {eff }}^{s-r e f}-\mathcal{E}_{\text {eff }}^{d}$, where $\mathcal{E}_{\text {eff }}^{d}$ indicates as before the Young's modulus associated with the design dimensions of the specimen. The repartition of molding accuracies $-1.2 \mathrm{kPa} \leq \Delta \mathcal{E}_{\text {eff }} \leq 0.6 \mathrm{kPa}$ is shown for $2 \mathrm{~L}$ (II) and $3 \mathrm{~L}$ (III) specimens by a boxplot in Fig. 9(b). Overall, $\Delta \mathcal{E}_{\text {eff }}$ is characterised by their mean plus minus their standard deviation as $\Delta \mathcal{E}_{\text {eff }}^{\mathrm{II}}=-0.03 \pm 0.43 \mathrm{kPa}$ for $2 \mathrm{~L}$ specimens and $\Delta \mathcal{E}_{\text {eff }}^{\mathrm{III}}=-0.43 \pm 0.81 \mathrm{kPa}$ for $3 \mathrm{~L}$ specimens. Thus, $\Delta \mathcal{E}_{\text {eff }}$ of the molded 2L specimens are distributed around a small mean value near $0 \mathrm{kPa}$ as $\pm 0.5 \mathrm{kPa}$ whereas $\Delta \mathcal{E}_{\text {eff }}$ of the molded 3L specimens are distributed around a negative mean value $(-0.20 \mathrm{kPa})$ as $-0.20 \pm 0.56 \mathrm{kPa}$. From the relative accuracies $\xi_{\mathcal{E}_{\text {eff }}}$ in Table 6 is seen that for all $2 \mathrm{~L}$ specimens $\left|\xi_{\mathcal{E}_{e f f}}\right| \leq 7.5 \%$ and for all 3L specimens $\left|\xi_{\mathcal{E}_{e f f}}\right| \leq 7.4 \%$. The absolute difference between modelled $\mathcal{E}_{\text {eff }}^{d}$ for designed specimens and $\mathcal{E}_{\text {eff }}^{s-r e f}$ for molded specimens remains limited to $\left|\Delta \mathcal{E}_{\text {eff }}\right| \leq 1.2 \mathrm{kPa}$. Thus molded specimens are suitable to validate the model and to assess potential influences on modelled $\mathcal{E}_{\text {eff }}$ such as stacking orientation, dimension ratio or composition ratio. 


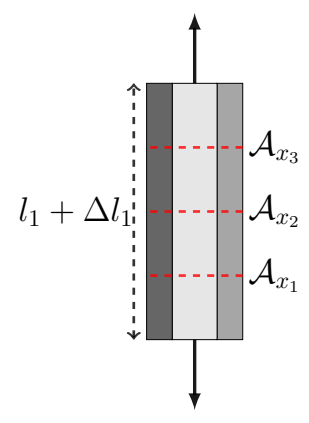

(a) parallel, $\|$

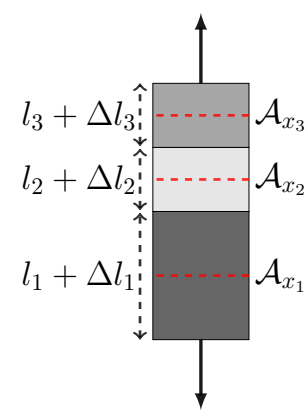

(b) serial, $\perp$

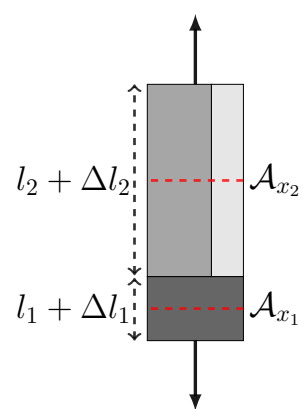

(c) combined, $\perp \|$

Figure 10: Measurement of layer lengths $l_{i}+\Delta l_{i}$ and cross-sectional areas $\mathcal{A}_{x}$. at different positions $x$. along the test section following an uni-axial force $\mathcal{F}$ (full arrows) on $3 \mathrm{~L}(n=3)$ specimens with different stacking: a) parallel $(\|)$ with $l_{1}+\Delta l_{1}=\ldots=l_{n}+\Delta l_{n}$, b) serial $(\perp)$, c) combined stacking $(\perp \|)$ with $l_{2}+\Delta l_{2}=l_{3}+\Delta l_{3}$.

\section{Uni-axial stress testing}

Two different stress test methods are used to induce stress $\sigma$ along the vertical $x$-axis either with a mechanical press (MP, section 6.2) or with precision loading (PL, section 6.3). Both methods result in experimental forceelongation curves $\mathcal{F}(\Delta l)$ as their elongation $\Delta l$ is obtained for different loads $\mathcal{F}(\mathrm{PL})$ or vice-versa $(\mathrm{MP})$ so that both methods can be cross-validated on the same specimens. Specimens are positioned vertically by clamping its end terminations depicted in Fig. 5(a), Regardless of the applied stress test, additional geometrical measurements are gathered as outlined in section 6.1.

\subsection{Geometrical measurements}

Geometrical measurements on 3L specimens with different stacking are illustrated in Fig. 10.

The length $l_{i}+\Delta l_{i}$ of each layer $i=1 \ldots n$ is measured (ruler with accuracy $1 \mathrm{~mm}$ ) for different loads $\mathcal{F}$ (or elongations $\Delta l$ ), where $l_{i}(i=1 \ldots n)$ denotes the initial layer length measured for the unloaded $(\mathcal{F}=0 \mathrm{~N})$ but vertically clamped specimen. As each clamped specimen is subjected to its own weight $(25.2 \pm 2.1 \mathrm{~g}), l_{i} \geq l_{i}^{s}$ holds, where $l_{i}^{s}$ indicates the layer length of the molded specimen. The sought total elongation $\Delta l$ of each specimen as a function of $\mathcal{F}$ is then obtained from the measured layer elongations $\Delta l_{i}$. 
For 1L specimens or ML specimens with parallel (\|) stacking (Fig. 10(a)), ${ }_{430} \Delta l=\Delta l_{1}$ holds since all layers have equal length regardless of $\mathcal{F}$. For ML specimens with serial $(\perp)$ stacking (Fig. 10(b) , $\Delta l=\sum_{i=1}^{n} \Delta l_{i}$ holds as the elongation of each layer depends on its molding composition and associated $\left(\mathcal{E}_{\text {eff }}\right)_{i}$. For 3L specimens with combined serial and parallel $(\perp \|)$ stacking (Fig. 10(c) $), \Delta l=\Delta l_{1}+\Delta l_{2}$ holds as parallel stacked layers $(i=2$ and $i=3$ ) have equal lengths for all $\mathcal{F}$.

The specimens cross-sectional area $\mathcal{A}$ perpendicular to the force or vertical $x$-direction is likely to reduce with $\mathcal{F}$ as schematically depicted in Fig. 3 . It follows that $\mathcal{A} \leq \mathcal{A}^{s}$ holds with $\mathcal{A}^{s}$ the initial cross-sectional area of the unloaded $(\mathcal{F}=0 \mathrm{~N})$ but vertically clamped specimen. The sought area $\mathcal{A}$ of each specimen is obtained from cross-sectional areas $\mathcal{A}_{x}$. (caliper Mitutoyo 500-196-30, accuracy $0.01 \mathrm{~mm}$ ) measured at two or three different vertical positions $x$. along its test section subjected to a constant load $(\mathcal{F} \geq 0 \mathrm{~N})$. For 1L specimens and ML specimens with parallel (\|) stacking (Fig. 10(a)), three area measurements $\mathcal{A}_{x_{1}}, \mathcal{A}_{x_{2}}$ and $\mathcal{A}_{x_{3}}$ are taken at $25 \%, 50 \%$ and $75 \%$ of the test section with length $l_{1}+\Delta l_{1}$. The sought cross-sectional area $\mathcal{A}$ is then obtained from their mean value,

$$
\mathcal{A}^{\|}=\frac{\mathcal{A}_{x_{1}}+\mathcal{A}_{x_{2}}+\mathcal{A}_{x_{3}}}{3}
$$

so that the measurement accuracy between different positions can be assessed. For serial $(\perp)$ stacked ML specimens (Fig. 10(b)), the cross-sectional area or each layer $\left(\mathcal{A}_{x_{i}}\right.$ with $\left.i=1 \ldots n\right)$ is measured midway. The sought cross-sectional area $\mathcal{A}$ is then obtained from the weighted arithmetic mean as

$$
\mathcal{A}^{\perp}=\frac{\sum_{i=1}^{n}\left(l_{i}+\Delta l_{i}\right) \mathcal{A}_{x_{i}}}{l+\Delta l} .
$$

For 3L specimens with combined serial and parallel $(\perp \|)$ stacking (Fig. 10(c) , two cross-sectional areas $\mathcal{A}_{x_{1}}$ and $\mathcal{A}_{x_{2}}$ are measured midway of the serial $(i=1)$ and of the parallel $(i=2$ or $i=3)$ stacked layers. The sought cross-sectional area $\mathcal{A}$ is thus given as the weighted arithmetic mean of the serial and one of the parallel layers (taken as $i=2$ ) as

$$
\mathcal{A}^{\perp \|}=\frac{\sum_{i=1}^{2}\left(l_{i}+\Delta l_{i}\right) \mathcal{A}_{x_{i}}}{l+\Delta l} .
$$




\subsection{Mechanical press}

$440 \quad$ An electro-mechanical press (3369, Instron Corp.) with $50 \mathrm{kN}$ force sensor (2530-445/71212, Instron Corp., accuracy 0.2\%) is used for uni-axial stress testing of specimens along the parallel $x$-axis (as depicted in Fig. 10) with typical forces $\mathcal{F}$ up to $8 \mathrm{~N}$. The mechanical press $(\mathrm{MP})$ was set for displacement control imposing four maximum elongations $\Delta l$ of $25,50,100$ and $150 \mathrm{~mm}$, respectively. The deformation rate was set to $1 \mathrm{~mm} / \mathrm{s}$ for the 25,50 and $100 \mathrm{~mm}$ elongations and to $2 \mathrm{~mm} / \mathrm{s}$ for the $150 \mathrm{~mm}$ elongation. Force and elongation time series (sampling rate of $10 \mathrm{~Hz}$ ) were measured during loading of the specimens so that for each specimen four MP datasets are obtained, i.e. one for each imposed maximum elongation. No plastic deformation was observed following their unloading (at $5 \mathrm{~mm} / \mathrm{s}$ ).

Due to the relative softness of the tested specimens ( $F \leq 8 \mathrm{~N}$ compared to the $50 \mathrm{kN}$ force sensor range), a moving-average filter with variable window size and 1 sample shift is applied to denoise the raw force sensor data in order to enhance the linearity in the force-elongation curves. Resulting smoothed MP force-elongation curves matches well with a linear fit of smoothed MP data as the coefficient of determination yields $R^{2} \geq 80 \%$ with a mean value of $88 \%$ and a standard deviation of $6 \%$. Raw and smooth MP datasets for maximum elongation $\Delta l=100 \mathrm{~mm}$ are illustrated in Fig. 11 for 2L specimens $\mathrm{II}_{2, \perp}$ and $\mathrm{II}_{3, \perp}$. Overall, the window size in terms of elongation ranges from $0.3 \mathrm{~mm}$ up to $6.5 \mathrm{~mm}$ (or 2 up to 65 samples), where larger window sizes are used for softer specimen (with lower $\mathcal{E}_{\text {eff }}$ ) as illustrated in Fig. 11 for $\mathrm{II}_{2, \perp}$ (window size $6.5 \mathrm{~mm}$ or 65 samples, modelled $\mathcal{E}^{\perp, s-r e f}=6.7 \mathrm{kPa}$ ) and $\mathrm{II}_{3, \perp}$ (window size $0.7 \mathrm{~mm}$ or 7 samples, modelled $\mathcal{E}^{\perp, s-r e f}=16.9 \mathrm{kPa}$ ). 465 The overall window elongation size corresponds to a mean plus minus standard deviation of $2.1 \pm 1.9 \mathrm{~mm}$ (or $19 \pm 17$ samples), which is in accordance with the constant window size of $2.0 \mathrm{~mm}$ (or 20 samples) proposed in [3] for $1 \mathrm{~L}$ specimens resulting in $\mathcal{E}_{\text {eff }}^{\text {ref }}$. In the following sections, smoothed MP force-elongation curves are considered.

Additional geometrical measurements described in section 6.1 are made for each clamped specimen without loading $(\Delta l=0 \mathrm{~mm})$ and once each of the maximum elongations is reached. Cross-sectional areas $\mathcal{A}$ of the specimens 


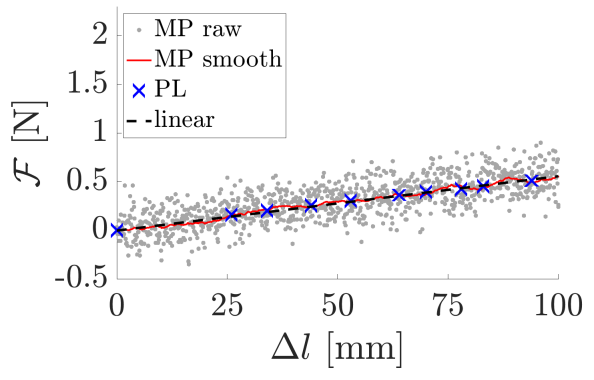

(a) $\mathrm{II}_{2, \perp}$

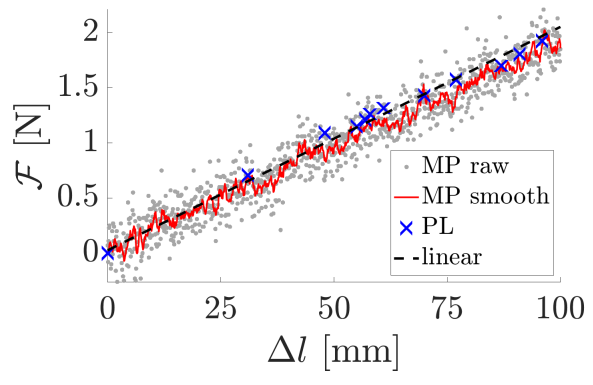

(b) $\mathrm{II}_{3, \perp}$

Figure 11: Linear behavior (dashed line) of measured force-elongation curves $\mathcal{F}(\Delta l)$ with the mechanical press (MP, raw and smooth) for maximum elongation $\Delta l=100 \mathrm{~mm}$ and with precision loading $(\mathrm{PL})$ for $2 \mathrm{~L}$ specimens: a) $\mathrm{II}_{2, \perp}$, modelled $\mathcal{E}^{\perp, s-r e f}=6.7 \mathrm{kPa}$, MP smooth with $6.5 \mathrm{~mm}$ (or 65 samples) window, PL for $m \leq 52 \mathrm{~g}, \mathrm{~b}$ ) $\mathrm{II}_{3, \perp}$, modelled $\mathcal{E}^{\perp, s-r e f}=16.9 \mathrm{kPa}$, MP smooth with $0.7 \mathrm{~mm}$ (or 7 samples) window, PL for $m \leq 196 \mathrm{~g}$.

are then obtained as outlined in section 6.1, so that $\mathcal{A}(\mathcal{F})$ contains 5 data points obtained at imposed maximum elongations $\Delta l \in\{0,25,50,100,150\} \mathrm{mm}$. Elongations $\Delta l$ deduced from geometrical measurements of layer lengths $l_{i}+\Delta l_{i}$ as outlined in section 6.1, matches the imposed maximum elongations to within $1 \mathrm{~mm}$ (or $\leq 4 \%$ difference for $\Delta l \geq 25 \mathrm{~mm}$ ), which corresponds to the ruler accuracy. It follows that the estimated accuracy of elongations $\Delta l$ obtained from geometrical measurements yields $\geq 96 \%$ for $\Delta l \geq 25 \mathrm{~mm}$. The accuracy increases with $\Delta l$.

\subsection{Precision loading}

Uni-axial stress testing (along the parallel $x$-axis) of a specimen by means of precision loading (PL) is performed by clamping its end terminations so that its upper end is fixed while a weight $m$ is added to the lower end. The weight is incremented with 5 up to $10 \mathrm{~g}$ (calibrated scale, Vastar $500 \mathrm{G} \mathrm{X}$ $0.01 \mathrm{G}$, accuracy $0.01 \mathrm{~g}$ ). The load force $\mathcal{F}$ for added mass $m$ yields $\mathcal{F}=m \cdot g_{0}$ with gravitational constant $g_{0}=9.81 \mathrm{~m} / \mathrm{s}^{2}$. For each weight increment, the specimens elongation $\Delta l$ is deduced from geometrical measurements of its layer lengths $l_{i}+\Delta l_{i}$, as outlined in section 6.1, with an estimated accuracy (section 6.2) of $\geq 96 \%$ for $\Delta l \geq 25 \mathrm{~mm}$. Depending on the specimen, the assessed total elongation varies between $55 \mathrm{~mm}$ and $255 \mathrm{~mm}$, corresponding to a total added weight between $71 \mathrm{~g}$ and $416 \mathrm{~g}$. The cross-sectional area $\mathcal{A}$ 


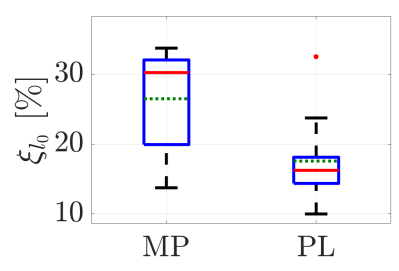

(a) $\xi_{l_{0}}$ at $\Delta l=0 \mathrm{~mm}$

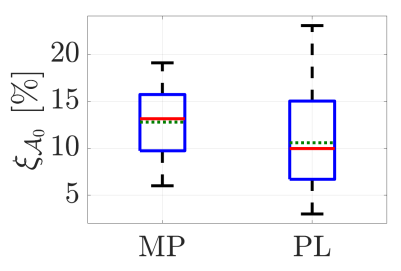

(b) $\xi_{\mathcal{A}_{0}}$ at $\Delta l=0 \mathrm{~mm}$

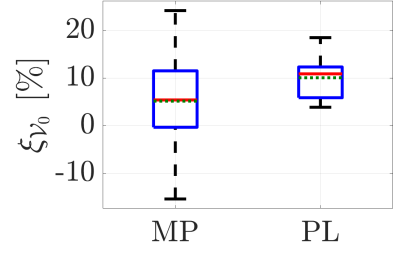

(c) $\xi_{\mathcal{V}_{0}}$ at $\Delta l=0 \mathrm{~mm}$

Figure 12: Boxplots with median (full line), mean (dotted line), interquartile range between the first and third quartile (box), extrema (whiskers) of the relative (\%) impact of clamping on the test section of molded specimens (superscript $s$ ) prior $(\Delta l=0 \mathrm{~mm})$ to testing with the mechanical press (MP) and with precision loading (PL): a) length $\left.\xi_{l_{0}}=\left(l_{0}-l^{s}\right) / l^{s}, \mathrm{~b}\right)$ cross-sectional area $\left.\xi_{\mathcal{A}_{0}}=\left(\mathcal{A}^{s}-\mathcal{A}_{0}\right) / \mathcal{A}^{s}, \mathrm{c}\right)$ volume $\xi_{\mathcal{V}_{0}}=\left(\mathcal{V}_{0}-\mathcal{V}^{s}\right) / \mathcal{V}^{s}$.

of tested specimens is measured as outlined in section 6.1 whenever the elongation increment due to added weights yields about $20 \pm 5 \mathrm{~mm}$ so that $\mathcal{A}(\mathcal{F})$ contains between 6 and 18 data points depending on the total elongation. A single PL force-elongation dataset per specimen is gathered without data smoothing as illustrated in Fig. 11 for $\mathrm{II}_{2, \perp}(m \leq 52 \mathrm{~g})$ and $\mathrm{II}_{3, \perp}(m \leq 196 \mathrm{~g})$. The PL force-elongation curves matches well with a linear PL data fit as the coefficient of determination yields $R^{2} \geq 90 \%$.

\section{Experimental results}

\subsection{Geometrical measurements on the test section}

\subsubsection{Initial test section geometry for clamped specimen at $\Delta l=0 \mathrm{~mm}$}

The geometry of the test section of unmounted molded specimens (superscript $s$ ) matches its design (superscript $d$, Fig. 5(a)) so that length $l^{s}=l^{d}$ $\left(l^{d}=80 \mathrm{~mm}\right)$, cross-sectional area $\mathcal{A}^{s}=\mathcal{A}^{d}\left(\mathcal{A}^{d}=150 \mathrm{~mm}^{2}\right)$ and volume $\mathcal{V}^{s}=\mathcal{V}^{d}\left(\mathcal{V}^{d}=12 \mathrm{~cm}^{3}\right)$. When specimens are mounted vertically by clamping its end terminations for uni-axial MP or PL testing, the geometry of the test section is affected. The influence of clamping on the geometry of the test section of mounted specimens is then obtained from geometrical measurements, illustrated in Fig. 10, at the origin of the force-elongation curves for $\Delta l=0 \mathrm{~mm}(\mathrm{MP})$ and $\mathcal{F}=0 \mathrm{~N}(\mathrm{PL})$. The geometry of the test section of clamped specimens at the origin (superscript 0) is then characterised by length $l_{0} \geq l^{s}$ and cross-sectional area $\mathcal{A}_{0} \leq \mathcal{A}^{s}$ so that its volume is obtained 
as $\mathcal{V}_{0}=l_{0} \cdot \mathcal{A}_{0}$. The relative discrepancy $\xi$. between geometrical test section characteristics of clamped and unmounted specimens is then quantified as:

$$
\begin{aligned}
\xi_{l_{0}} & =\frac{l_{0}-l^{s}}{l^{s}}, \\
\xi_{\mathcal{A}_{0}} & =\frac{\mathcal{A}^{s}-\mathcal{A}_{0}}{\mathcal{A}^{s}}, \\
\xi_{\mathcal{V}_{0}} & =\frac{\mathcal{V}_{0}-\mathcal{V}^{s}}{\mathcal{V}^{s}} .
\end{aligned}
$$

The repartition of these initial geometrical discrepancies due to clamping at $\Delta l=0 \mathrm{~mm}$ and $\mathcal{F}=0 \mathrm{~N}$ is represented by boxplots in Fig. 12. Overall, clamping of the specimens results in an increase of their length $l_{0}$ as $14 \% \leq \xi_{l_{0}} \leq 34 \%$ and an associated decrease of their cross-sectional area $\mathcal{A}_{0}$ as $6 \% \leq \xi_{\mathcal{A}_{0}} \leq 16 \%$ for both MP and PL tests. The magnitude of $\xi_{l_{0}}$ and $\xi_{\mathcal{A}_{0}}$ depends on the elasticity of the specimen as $l_{0}$ and $\mathcal{A}_{0}$ vary so that volume conservation applies to the test section since $\mathcal{V}_{0}=12.6 \pm 0.8 \mathrm{~cm}^{3}$ for $\mathrm{MP}$ and $\mathcal{V}_{0}=13.2 \pm 0.4 \mathrm{~cm}^{3}$ for PL. Thus, $\mathcal{V}_{0}$ varies little $(\leq 6 \%)$ between specimens and between test methods MP and PL. This is also seen from 515 the overall repartition of $\xi_{\mathcal{V}_{0}}$ in Fig. $12(\mathrm{c})$ for which the median and mean match to within $1 \%$ and the variation is mostly contained to within $6 \%$ of the medians. It is noted that the volume associated with the test section of the mounted molded specimens is increased up to $\xi_{\mathcal{V}_{0}} \leq 12 \%$ compared to its design value $\mathcal{V}^{d}=12 \mathrm{~cm}^{3}$.

\subsubsection{Area of the test section of specimens at $\Delta l \geq 0 \mathrm{~mm}$}

Geometrical measurements of the test sections cross-sectional areas $\left(\mathcal{A}_{x_{1}}\right.$, $\mathcal{A}_{x_{2}}, \mathcal{A}_{x_{3}}$ depicted in Fig. 10 at different $x$-positions along the force direction are made for at least 5 different elongations $\Delta l$ (or points along $\mathcal{F}(\Delta l)$ ) during uni-axial MP or PL stress testing (section 6.1). For each elongation $\Delta l$, the cross-sectional area $\mathcal{A}$ of the test section is then obtained as either $\mathcal{A}=\mathcal{A}^{\|}$(Eq. (9) $), \mathcal{A}=\mathcal{A}^{\perp}$ (Eq. (10)) or $\mathcal{A}=\mathcal{A}^{\perp \|}$ (Eq. (11)) depending on the stacking orientation of the specimen. It follows that geometrical data of the specimens cross-sectional area as a function of its elongation $\mathcal{A}(\Delta l)$ are gathered. Geometrical measurements $\left(\mathcal{A}_{x_{1}}, \mathcal{A}_{x_{2}}, \mathcal{A}_{x_{3}}\right)$ and resulting $\mathcal{A}$ for a parallel $\left(\mathrm{II}_{2, \|}\right)$ and serial $\left(\mathrm{III}_{3, \perp}\right)$ stacked specimen subjected to $\mathrm{PL}$ testing are illustrated in Fig. 13(a) and Fig 13(b), respectively. Matching (difference 


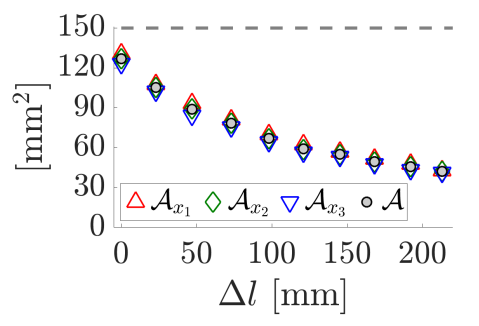

(a) $\mathrm{II}_{2, \|}-\mathrm{PL}$

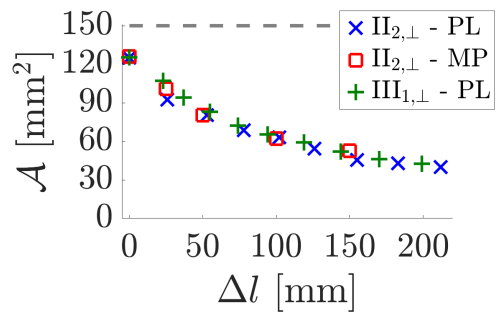

(c) $\mathrm{II}_{2, \perp}$ and $\mathrm{III}_{1, \perp}$

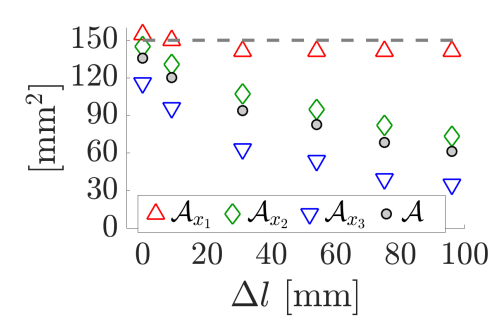

(b) $\mathrm{III}_{3, \perp}-\mathrm{PL}$

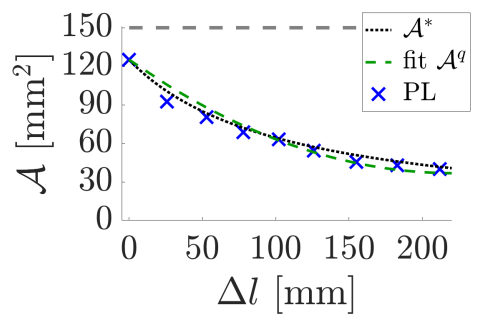

(d) $\mathrm{II}_{2, \perp}-\mathrm{PL}$

Figure 13: Cross-sectional area measurements $\left(\mathcal{A}_{x_{1}}, \mathcal{A}_{x_{2}}, \mathcal{A}_{x_{3}}\right)$ and specimens area $\mathcal{A}$ at different elongations $\Delta l$ during uni-axial MP and PL testing. Approximations from quadratic data fitting $\mathcal{A}^{q}(\Delta l)$ and volume conservation $\mathcal{A}^{*}(\Delta l)$ : a) $\mathcal{A}_{x_{1}}, \mathcal{A}_{x_{2}}$ and $\mathcal{A}_{x_{3}}$ for $\mathrm{II}_{2, \|}$ (PL test), b) $\mathcal{A}_{x_{1}}, \mathcal{A}_{x_{2}}$ and $\mathcal{A}_{x_{3}}$ for $\left.\mathrm{III}_{3, \perp}(\mathrm{PL}), \mathrm{c}\right) \mathcal{A}(\Delta l)$ for $\mathrm{II}_{2, \perp}$ (PL and $\mathrm{MP}$ ) and $\left.\mathrm{III}_{1, \perp}(\mathrm{PL}), \mathrm{d}\right) \mathcal{A}(\Delta l)$ for $\mathrm{II}_{2, \perp}(\mathrm{PL})$, fit $\mathcal{A}^{q}(\Delta l)\left(R^{2}=99 \%\right)$ and volume conservation $\mathcal{A}^{*}(\Delta l)\left(R^{2}=99 \%\right)$. Design value $\mathcal{A}^{d}=150 \mathrm{~mm}^{2}$ (horizontal dashed line) is indicated. 
less than $5 \mathrm{~mm}^{2}$ ) area measurements $\mathcal{A}_{x_{1}}(\Delta l), \mathcal{A}_{x_{2}}(\Delta l)$ and $\mathcal{A}_{x_{3}}(\Delta l)$ along the parallel stacked specimen $\mathrm{II}_{2, \|}$ in Fig. $13(\mathrm{a})$ illustrate the accuracy and repeatability of these area measurements and resulting $\mathcal{A}(\Delta l)$ data. The accuracy of $\mathcal{A}(\Delta l)$ is further illustrated in Fig. 13(c) as plotted $\mathcal{A}(\Delta l)$ data obtained during MP and PL testing of the same specimen $\mathrm{II}_{2, \perp}$ match (difference less than $\left.9 \mathrm{~mm}^{2}\right)$. Moreover, $\mathcal{A}(\Delta l)$ data obtained for specimens with a similar overall composition overlap as shown for $\mathrm{II}_{2, \perp}$ and $\mathrm{III}_{1, \perp}$ subjected to PL testing in Fig. 13(c).

A quadratic fit is applied to the measured $\mathcal{A}(\Delta l)$ data for each specimen resulting in a continuous approximation $\mathcal{A}^{q}(\Delta l)$. The non-zero constant in the quadratic fit is set to match $\mathcal{A}_{0}$, e.e. the initial value of $\mathcal{A}$ for the clamped specimen at $\Delta l=0 \mathrm{~mm}$ (section 7.1.1). As volume conservation applies to the test section during deformation, an alternative expression for the area $\mathcal{A}^{*}(\Delta l)$ is obtained using initial values $l_{0}$ and $\mathcal{A}_{0}$ at $\Delta l=0 \mathrm{~mm}$ as

$$
\mathcal{A}^{*}(\Delta l)=\frac{\mathcal{A}_{0} l_{0}}{l_{0}+\Delta l}
$$

Consequently, $\mathcal{A}^{*}$ requires no geometrical measurements other than $l_{0}$ and $\mathcal{A}_{0}$ whereas the quadratic fit requires geometrical measurements at different $\Delta l \geq 0 \mathrm{~mm}$. Area approximations $\mathcal{A}^{q}(\Delta l)$ (fit) and $\mathcal{A}^{*}(\Delta l)($ Eq. (13)) are plotted in Fig. 13(d) for specimen $\mathrm{II}_{2, \perp}$ subjected to PL testing. Both, the quadratic fit $\mathcal{A}^{q}(\Delta l)$ and volume conservation $\mathcal{A}^{*}(\Delta l)$ provide accurate estimations of $\mathcal{A}(\Delta l)$. The coefficient of determination for $\mathcal{A}^{q}(\Delta l)$ with respect to $\mathcal{A}(\Delta l)$ yields $R^{2} \geq 94 \%$. The coefficient of determination for $\mathcal{A}^{*}(\Delta l)$ with respect to $\mathcal{A}(\Delta l)$ yields $R^{2} \geq 84 \%$ for $1 \mathrm{~L}$ specimens and $R^{2} \geq 92 \%$ for $2 \mathrm{~L}$ and 3L specimens. This shows that areas $\mathcal{A}$ deduced from measured values $\mathcal{A}_{x}$. with Eq. (9), Eq. (10) or Eq. (11) provide realistic values for each stacking.

\subsection{Young's modulus estimation}

Force-elongation curves $\mathcal{F}(\Delta l)$ and geometrical test section characteristics obtained during MP or PL testing allow to obtain experimental stress-strain curves for each tested specimen. As the test sections geometry was shown to vary (e.g. cross-sectional area $\mathcal{A}$ in section 7.1.2), the true stress $\sigma_{t}$ and true strain $\varepsilon_{t}$ are assessed. The true stress $\sigma_{t}$ is then obtained as in Eq. (1) using 


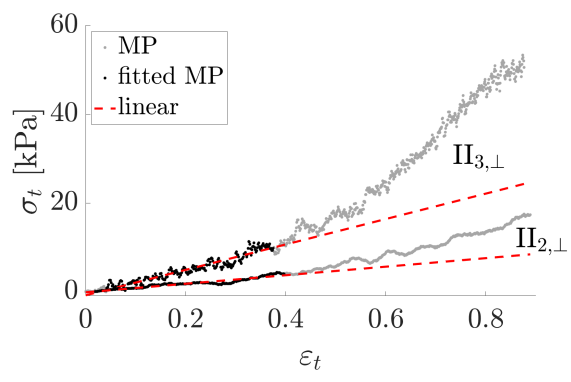

(a) $\mathrm{II}_{2, \perp}$ and $\mathrm{II}_{3, \perp}-\mathrm{MP}$

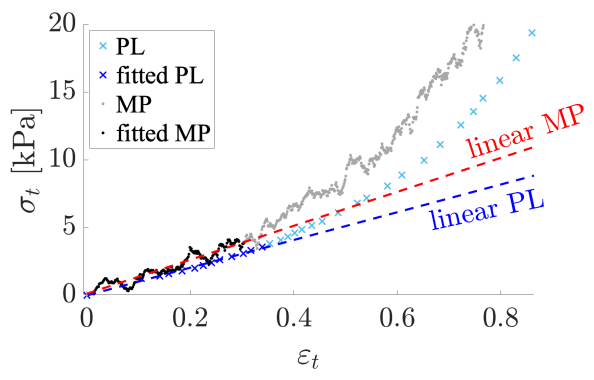

(b) $\mathrm{II}_{2, \|}-\mathrm{MP}$ and $\mathrm{PL}$

Figure 14: Examples of stress-strain curves $\sigma_{t}\left(\varepsilon_{t}\right)$ from MP $(\bullet)$ or PL $(\times)$ testing, data within the linear elastic region (fitted MP or PL) and linear fit (dashed line) whose slope $\left(R^{2} \geq 90 \%\right)$ corresponds to the elastic Young's modulus $\mathcal{E}_{\text {eff }}^{M P}$ or $\mathcal{E}_{\text {eff }}^{P L}$ : a) MP results for specimens $\mathrm{II}_{2, \perp}$ and $\mathrm{II}_{3, \perp}$, b) MP and PL results for specimen $\mathrm{II}_{2, \|}$.

instantaneous area $\mathcal{A}^{q}$ (or alternatively $\mathcal{A}^{*}$ ) so that

$$
\sigma_{t}=\frac{\mathcal{F}}{\mathcal{A}^{q}}
$$

Similarly, the true strain $\varepsilon_{t}=\int \frac{\delta l}{l}$ is obtained using instantaneous length $l$ so that

$$
\varepsilon_{t}=\ln \left(\frac{l}{l_{0}}\right) .
$$

The experimental elastic Young's modulus of each specimen from either MP $\left(\mathcal{E}_{\text {eff }}^{M P}\right)$ or PL $\left(\mathcal{E}_{\text {eff }}^{P L}\right)$ testing is then obtained as the slope of a linear fit to the elastic region in which stress $\sigma_{t}$ is proportional to the strain $\varepsilon_{t}$ so that

$$
\mathcal{E}_{\text {eff }}=\frac{\sigma_{t}}{\varepsilon_{t}}
$$

in accordance with Hooke's law of linear elastic deformation. The elastic 555 region $0 \leq \varepsilon_{t} \leq 0.2$ is extended to $\varepsilon_{t}>0.2$ as long as the linear fit accuracy $R^{2}$ increases until at least $R^{2} \geq 90 \%$. The mean and standard deviation of the overall upper limit of the linear region yields $\varepsilon_{t}=0.3 \pm 0.1$ which corresponds to an elongation of $31 \pm 9 \mathrm{~mm}$ for PL testing $\left(R^{2} \geq 97 \%\right)$ and an elongation 


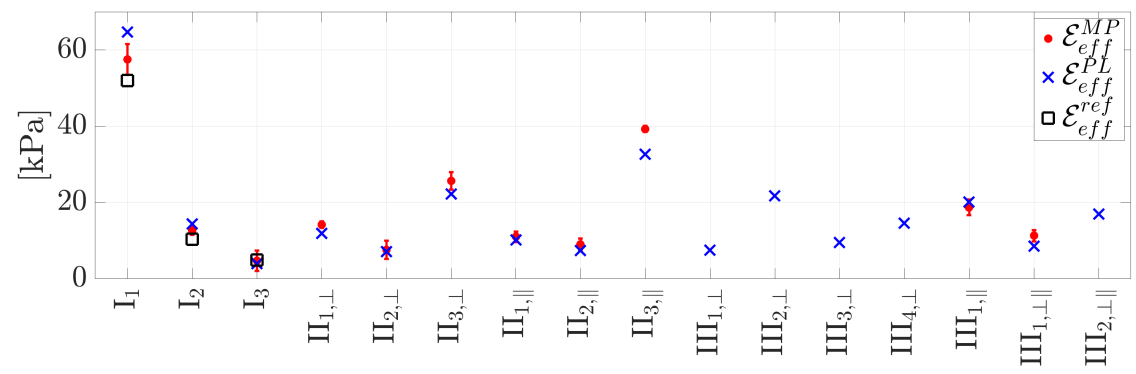

Figure 15: Young's moduli $\mathcal{E}_{\text {eff }}^{M P}\left(\right.$ mean $(\bullet)$ and standard deviation (bar)) and $\mathcal{E}_{\text {eff }}^{P L}(\times)$ for $1 \mathrm{~L}(\mathrm{I}),. 2 \mathrm{~L}$ (II.) and 3L (III.) specimens. $\mathcal{E}_{\text {eff }}^{r e f}$ from Table 3 ([3, 8] ) are plotted for I..

Table 7: 1L specimen and single layer composition: reference $\mathcal{E}_{\text {eff }}^{r e f}$ [3, 8] and measured $\mathcal{E}_{\text {eff }}^{M P}$ and $\mathcal{E}_{\text {eff }}^{P L}$ from MP and PL tests.

\begin{tabular}{c||c||c|c}
\hline Specimen & $\mathcal{E}_{\text {eff }}^{\text {ref }}[\mathrm{kPa}]$ & $\mathcal{E}_{\text {eff }}^{M P}[\mathrm{kPa}]$ & $\mathcal{E}_{\text {eff }}^{P L}[\mathrm{kPa}]$ \\
\hline \hline $\mathrm{I}_{1}$ & 52.0 & 57.5 & 64.7 \\
$\mathrm{I}_{2}$ & 10.4 & 12.7 & 14.4 \\
$\mathrm{I}_{3}$ & 4.9 & 4.7 & 4.0 \\
\hline
\end{tabular}

of $29 \pm 13 \mathrm{~mm}$ for MP testing $\left(R^{2} \geq 90 \%\right)$. Examples of experimental MP and PL stress-strain curves, their linear elastic regions and associated linear stress-strain data fits are illustrated in Fig. 14. Sought slopes $\mathcal{E}_{\text {eff }}^{M P}$ and $\mathcal{E}_{\text {eff }}^{P L}$ for each of the tested specimen are plotted in Fig. 15. For each of the MP tested specimens, the mean and standard deviation $(\leq 4.1 \mathrm{kPa})$ are plotted as four values are obtained, one for each of the imposed maximum elongations ${ }_{565} \Delta l \in\{25,50,100,150\} \mathrm{mm}$. For the $1 \mathrm{~L}$ specimens (I.), literature values $\mathcal{E}_{\text {eff }}^{\text {ref }}$ reported in Table 3 [3, 8] are plotted as well.

Values for $1 \mathrm{~L}$ specimens (I.) $\mathcal{E}_{\text {eff }}^{r e f}, \mathcal{E}_{\text {eff }}^{M P}$ and $\mathcal{E}_{\text {eff }}^{P L}$ are detailed in Table 7 . Although reference values $\mathcal{E}_{\text {eff }}^{\text {ref }}$ are of the same order or magnitude as $\mathcal{E}_{\text {eff }}^{M P}$ 570 and $\mathcal{E}_{\text {eff }}^{P L}$ for all three specimens, $\mathcal{E}_{\text {eff }}^{\text {ref }}$ underestimates measured $\mathcal{E}_{\text {eff }}^{M P}$ and $\mathcal{E}_{\text {eff }}^{P L}$ for $\mathrm{I}_{1}$ (with $5.5 \mathrm{kPa}$ for $\mathrm{MP}$ and $12.7 \mathrm{kPa}$ for $\mathrm{PL}$ ) and for $\mathrm{I}_{2}$ (with $2.3 \mathrm{kPa}$ for $\mathrm{MP}$ and $4 \mathrm{kPa}$ for $\mathrm{PL}$ ) whereas all $\mathcal{E}_{\text {eff }}$-values (MP, PL and reference from literature) match to within $1 \mathrm{kPa}$ for $\mathrm{I}_{3}$. Therefore, model validation for molded ML specimens in section 8 is assessed using layer values given 575 by $\mathcal{E}_{\text {eff }}^{r e f}$ (as for the modelled design values of molded ML specimens $\mathcal{E}_{\text {eff }}^{s-r e f}$ 


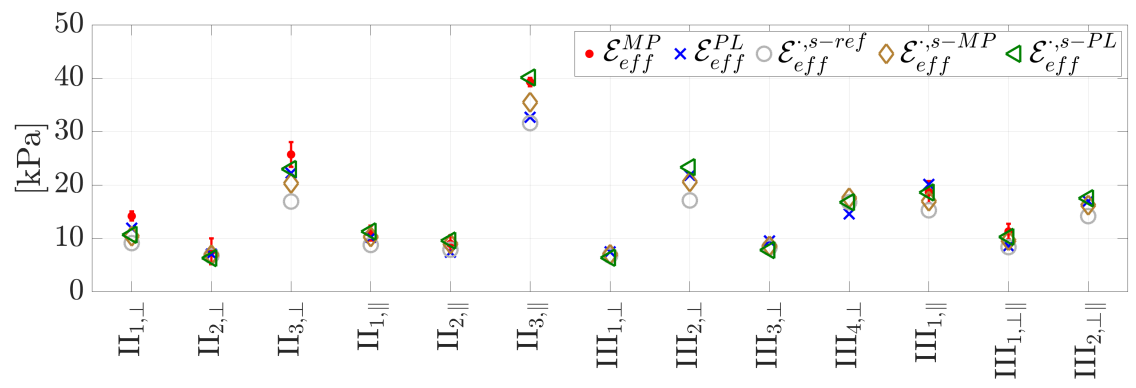

Figure 16: Measured and modelled $\mathcal{E}_{\text {eff }}$ for 2L (II.) and 3L (III.) specimens.

in Table 6 and by the measured $\mathcal{E}_{\text {eff }}^{M P}$ and $\mathcal{E}_{\text {eff }}^{P L}$ for which modelled values are denoted $\mathcal{E}_{\text {eff }}^{s-M P}$ and $\mathcal{E}_{\text {eff }}^{s-P L}$, respectively. It is further noted that since all 2L (II.) and 3L (III.) specimens are composed of a combination of the composition of the $1 \mathrm{~L}$ specimens, all measured $\mathcal{E}_{\text {eff }}^{M P}$ and $\mathcal{E}_{\text {eff }}^{P L}$ are within the range spanned between the softest $\left(I_{3}\right)$ and most rigid $\left(I_{1}\right) 1 \mathrm{~L}$ specimen.

The mean and standard deviation of the overall difference between mean $\mathcal{E}_{\text {eff }}^{P L}$ and $\mathcal{E}_{\text {eff }}^{M P}$ for all specimens yields $-0.8 \pm 3.5 \mathrm{kPa}$. Thus the overall difference is of the same order of magnitude as the standard deviation $\leq 4.1 \mathrm{kPa}$ observed between different MP tests on the same specimen so that both MP and PL tests provide accurate measurements of $\mathcal{E}_{\text {eff }}$ for all specimens. Therefore, most of the 3L specimens are subjected only to PL testing as PL testing provides the highest fit accuracy $\left(R^{2} \geq 97 \%\right)$ of the linear elastic region with slope $\mathcal{E}_{\text {eff }}^{P L}$. Obtained $\mathcal{E}_{\text {eff }}^{P L}$ for $2 \mathrm{~L}$ and $3 \mathrm{~L}$ specimens with similar compositions match as the difference is limited to $0.4 \mathrm{kPa}$ between $\mathrm{II}_{2, \perp}$ and $\mathrm{III}_{1, \perp}$ and to $-0.4 \mathrm{kPa}$ between $\mathrm{II}_{3, \perp}$ and $\mathrm{III}_{2, \perp}$. This confirms the model assumption that changing the layer order, in this case due to layer splitting and layer permutation, in ML specimens does not affect the Young's modulus when the overall composition remains similar.

\section{Modelled Young's modulus validation for ML specimens}

The effective Young's modulus for each of the molded ML specimens is modelled as outlined in section 3 while the stacking composition ratio $\mathcal{B}_{j, j+1}$ between adjacent layers is calculated using the single layer compositions $\mathcal{E}_{\text {eff }}$ associated with 1L specimens summarised in Table 7. Thus layer compo- 
sitions are either taken from literature $\left(\mathcal{E}_{\text {eff }}^{r e f}\right)$ as during specimens design (Table 6), or obtained from the measured MP $\left(\mathcal{E}_{\text {eff }}^{M P}\right)$ and $\mathrm{PL}\left(\mathcal{E}_{\text {eff }}^{P L}\right)$ tests on 1L specimens. The corresponding modelled effective Young's modulus of the homogeneous elastic specimen yields $\mathcal{E}_{\text {eff }}^{\cdot, s-r e f}, \mathcal{E}_{\text {eff }}^{, s-M P}$ and $\mathcal{E}_{\text {eff }}^{, s-P L}$, respectively. Modelled $\mathcal{E}_{\text {eff }}^{, s-r e f}, \mathcal{E}_{\text {eff }}^{, s-M P}$ and $\mathcal{E}_{\text {eff }}^{, s-P L}$ and measured $\mathcal{E}_{\text {eff }}^{M P}$ and $\mathcal{E}_{\text {eff }}^{P L}$ for ML specimens (II. and III.) are plotted in Fig. 16. Overall measured and modelled values are of the same order of magnitude so that the intended variation - reflecting the impact of stacking orientation, stacking dimension ratios and stacking composition underlying the ML specimens design - is observed for both the measured and modelled $\mathcal{E}_{\text {eff }}$.

The repartitions of the differences between the measured $\mathcal{E}_{\text {eff }}^{P L}$ for each ML specimen and each of the model values $\mathcal{E}_{\text {eff }}^{, s-r e f}, \mathcal{E}_{\text {eff }}^{, s-M P}$ and $\mathcal{E}_{\text {eff }}^{, s-P L}$ is assessed by boxplots in Fig. 17. As a comparison, also the difference between measured $\mathcal{E}_{\text {eff }}^{P L}$ and measured $\mathcal{E}_{\text {eff }}^{M P}$ for ML specimens subjected to both PL and MP testing is shown as well. It is noted that model differences and measured differences are of the same order of magnitude so that the model approach is validated. In Fig. 16 is seen that $\mathcal{E}_{\text {eff }}^{P L}$ is slightly underestimated by $\mathcal{E}_{\text {eff }}^{, s-r e f}$, so that the mean $(1.7 \mathrm{kPa})$ and median $(1.2 \mathrm{kPa})$ differences ${ }_{620}$ with respect to $\mathcal{E}_{\text {eff }}^{,, s-r e f}$ are positive. The overall model accuracy improves for $\mathcal{E}_{\text {eff }}^{, s-M P}$ and for $\mathcal{E}_{\text {eff }}^{,, s-P L}$ compared to $\mathcal{E}_{\text {eff }}^{, s-r e f}$ as the range spanned between the extrema reduces from $\left[\begin{array}{ll}-2.2 & 5.3\end{array}\right] \mathrm{kPa}$ to within $\left[\begin{array}{ll}-3.0 & 3.0\end{array}\right] \mathrm{kPa}$ for $\mathcal{E}_{e f f}^{, s-M P}$ and even further to within $\left[\begin{array}{ll}-2.2 & 1.7\end{array}\right] \mathrm{kPa}$ for $\mathcal{E}_{\text {eff }}^{\cdot, s-P L}$. Consequently, the overall model accuracy in terms of these extrema yields $\pm 3 \mathrm{kPa}$ for $\mathcal{E}_{\text {eff }}^{, s-M P}$ and $\pm 2.2 \mathrm{kPa}$ for $\mathcal{E}_{\text {eff }}^{, s-P L}$. Both repartitions of the differences between $\mathcal{E}_{\text {eff }}^{P L}$ and either $\mathcal{E}_{\text {eff }}^{, s-M P}$ or $\mathcal{E}_{\text {eff }}^{, s-P L}$ are characterised by a mean and median value near zero $(<1 \mathrm{kPa})$ and a standard deviation which is less than $\pm 2.4 \mathrm{kPa}$, which is the same as the standard deviation obtained for the difference between experimental values $\mathcal{E}_{\text {eff }}^{P L}$ and $\mathcal{E}_{\text {eff }}^{M P}$. Consequently, the overall model accuracy in terms of the standard deviation yields $\pm 2.4 \mathrm{kPa}$. It is noted that for the soft specimen with $\mathcal{E}_{\text {eff }}^{P L} \leq 15 \mathrm{kPa}$, this accuracy increases to $\pm 1.5 \mathrm{kPa}$. 


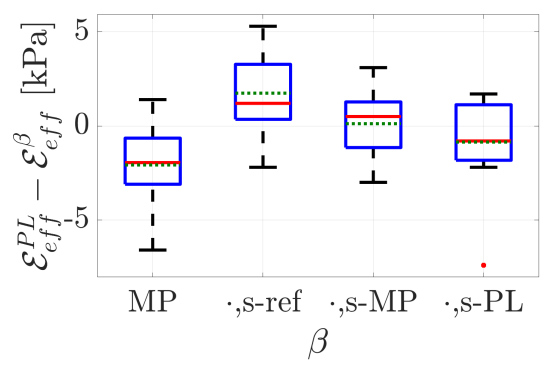

Figure 17: Boxplots with median (full line), mean (dotted line), interquartile range between the first and third quartile (box), extrema (whiskers) of the overall difference for ML molded specimens between $\mathcal{E}_{\text {eff }}^{P L}$ from PL tests and $\mathcal{E}_{\text {eff }}^{\beta}$ set to: a) measured $\mathcal{E}_{\text {eff }}^{M P}$ from MP tests, b) modelled $\mathcal{E}_{\text {eff }}^{\cdot, s-r e f}$, c) modelled $\mathcal{E}_{\text {eff }}^{, s-M P}$, d) modelled $\mathcal{E}_{\text {eff }}^{,, s-P L}$.

\section{Conclusion}

Sixteen silicone-molded 1L, 2L or 3L specimens are considered. ML specimens were designed so that the stacking orientation (serial, parallel or combined) and the range of composition and dimension ratios affect the specimens effective elastic Young's modulus $\mathcal{E}_{\text {eff }}$. Concretely, the specimens $\mathcal{E}_{\text {eff }}$ varies between $4 \mathrm{kPa}$ and $65 \mathrm{kPa}$, which overlaps the range associated with normal human VF's (up to $60 \mathrm{kPa}$ ). The $\mathcal{E}_{\text {eff }}$ of six $2 \mathrm{~L}$ and two $3 \mathrm{~L}$ specimens is experimentally determined from MP and from PL testing so that both methods are cross-validated as their difference yields less than $3.5 \mathrm{kPa}$, which is of the same order of magnitude as the difference $(\leq 4.1 \mathrm{kPa})$ associated with multiple MP testing on the same specimen. An analytical model of $\mathcal{E}_{\text {eff }}$ for ML specimens composed of serial and/or parallel stacked layers is then validated against the measured $\mathcal{E}_{\text {eff }}$ from PL testing on six $2 \mathrm{~L}$ and seven $3 \mathrm{~L}$ molded specimens. The overall model accuracy yields $\pm 2.4 \mathrm{kPa}$. The composition and dimension of each layer are designed so that stacking composition ratios $(0.2 \leq \mathcal{B} \leq 5)$ and stacking dimension ratios $(0.11 \leq \nabla \leq 6.6)$ of adjacent layers are pertinent to ML representations of human VF's. Future research is needed to validate the proposed model on silicone-molded VF replicas mimicking the ML structure of a human VF. Such a validation would contribute to the systematic study of the influence of the effective Young's modulus on the auto-oscillation of silicone-molded VF replicas. The dimensional overall molding accuracy of all serial and parallel molded layers yields $\pm 1.5 \mathrm{~mm}$. As this dimensional molding accuracy affects $\mathcal{E}_{\text {eff }}$ with less than $1.2 \mathrm{kPa}$, the molding of the specimens is considered reproducible. 


\section{Acknowledgements}

${ }_{660}$ This work was partly supported by Full3DTalkingHead project (ANR20-CE23-0008-03) and a PhD grant from the French Ministry of Education and Research. Authors thank Prof. G. Combe and F. Girard (Grenoble INP, France) for the usage of the electro-mechanical press.

\section{References}

[1] B. Pickup, S. Thomson, Flow-induced vibratory response of idealized versus magnetic resonance imaging-based synthetic vocal fold models, J Acous Soc Am 128 (2010) 124-129.

[2] P. Murray, S. Thomson, Vibratory responses of synthetic, self-oscillating vocal fold models, J Acous Soc Am 132 (2012) 3428-3438.

[3] A. Bouvet, Experimental and theoretical contribution to the analysis and the modelling of the vocal folds vibration, Ph.D. thesis, Grenoble Alpes University, France, 2019.

[4] I. Tokuda, R. Shimamura, Effect of level difference between left and right vocal folds on phonation: Physical experiment and theoretical study, J Acous Soc Am 142 (2017) 482-492.

[5] C. Rosen, C. Simpson, Operative techniques in laryngology, SpringerVerlag, 2008.

[6] M. Hirano, Clinical examination of voice, Springer-Verlag, 1981.

[7] A. Bouvet, X. Pelorson, A. Van Hirtum, Influence of water spraying on an oscillating channel, J. Fluids and Structures 93 (2020) 1-20.

[8] A. Bouvet, I. Tokuda, X. Pelorson, A. Van Hirtum, Influence of level difference due to vocal folds angular asymmetry on auto-oscillating replicas, J. Acoust. Soc. 147 (2020) 1136-1145.

[9] R. Scherer, D. Shinwari, K. De Witt, C. Zhang, B. Kucinschi, A. Afjeh, Intraglottal pressure profiles for a symmetric and oblique glottis with a divergence angle of 10 degrees, J Acous Soc Am 109 (2001) 1616-1630. 
[10] G. Berke, B. Gerratt, Laryngeal biomechanics: an overview of mucosal wave mechanics, J Voice 7 (1993) 123-128.

[11] T. Riede, C. Brown, Body size, vocal fold length, and fundamental frequency: implications for mammal vocal communication, Nova Acta Leopoldina NF 111380 (2013) 295-314.

[12] M. Hirano, S. Kurita, T. Nakashima, Vocal fold physiology: contempory research and clinical issues, College-Hill Press, 1983, pp. 22-43.

[13] F. Alipour, I. Titze, Elastic models of vocal fold tissues, J Acous Soc Am 90 (1991) 1326-1331.

[14] Y. Min, I. Titze, F. Alipour, Stress-strain response of the human vocal ligament, Ann Otol Rhinol Laryngol 104 (1995) 563-569.

[15] R. Chan, M. Fu, L. Young, N. Tirunagari, Relative contributions of collagen and elastin to elasticity of the vocal fold under tension, Ann Biomed. Eng. 35 (2007) 1471-1483.

[16] S. Smith, S. Thomson, Effect of inferior surface angle on the selfoscillation of a computational vocal fold model, J Acous Soc Am 131 (2012) 4062-4075.

[17] D. Chhetri, Z. Zhang, J. Neubauer, Measurement of Young's modulus of vocal folds by indentation, J Voice 25 (2011) 1-7.

[18] A. Miri, Mechanical characterization of vocal fold tissue: a review study, J Voice 28 (2014) 657-666.

[19] Z. Zhang, H. Samajder, J. Long, Biaxial mechanical properties of human vocal fold cover under fold elongation, J Acoust Soc Am 29 (2017) EL356.

[20] J. Drechsel, S. Thomson, Influence of supraglottal structures on the glottal jet exiting a two-layer synthetic, self-oscillating vocal fold model, J. Acous. Soc. Am. 123 (2008) 4434-4445.

[21] T. Riede, I. Tokuda, J. Munger, S. Thomson, Mammalian laryngseal air sacs add variability to the vocal tract impedance: Physical and computational modeling, J Acous Soc Am 124 (2008) 634-647. 
[22] P. Murray, S. Thomson, Synthetic, multi-layer, self-oscillating vocal fold model fabrication, J Vis Exp 58 (2011) e3498.

[23] Smooth-On, Material description, 2020. URL: https://www. smooth-on.com/products/.

[24] W. Voigt, Ueber die beziehung zwischen den beiden elasticitätsconstanten isotroper körper, Ann Phys 274 (1889) 573-587.

[25] A. Reuss, Berechnung der fließgrenze von mischkristallen auf grund der plastizitätsbedingung für einkristalle, ZAMM 9 (1929) 49-58. 